\title{
NLK phosphorylates Raptor to mediate stress-induced mTORC1 inhibition
}

\author{
Hai-Xin Yuan, ${ }^{1,3}$ Zhen Wang, ${ }^{1}$ Fa-Xing Yu, ${ }^{2,3}$ Fulong Li, ${ }^{1}$ Ryan C. Russell, ${ }^{3}$ Jenna L. Jewell, ${ }^{3}$ \\ and Kun-Liang Guan ${ }^{3}$ \\ ${ }^{1}$ Key Laboratory of Molecular Medicine of Ministry of Education, Institutes of Biomedical Sciences, Shanghai Medical College, \\ Fudan University, Shanghai 20032, China; ${ }^{2}$ Children's Hospital, Institutes of Biomedical Sciences, Fudan University, Shanghai \\ 20032, China; ${ }^{3}$ Department of Pharmacology and Moores Cancer Center, University of California at San Diego, La Jolla, California \\ 92130, USA
}

The mechanistic target of rapamycin (mTOR) is a central cell growth controller and forms two distinct complexes: mTORC1 and mTORC2. mTORC1 integrates a wide range of upstream signals, both positive and negative, to regulate cell growth. Although mTORC1 activation by positive signals, such as growth factors and nutrients, has been extensively investigated, the mechanism of mTORC1 regulation by stress signals is less understood. In this study, we identified the Nemo-like kinase (NLK) as an mTORC1 regulator in mediating the osmotic and oxidative stress signals. NLK inhibits mTORC1 lysosomal localization and thereby suppresses mTORC1 activation. Mechanistically, NLK phosphorylates Raptor on S863 to disrupt its interaction with the Rag GTPase, which is important for mTORC1 lysosomal recruitment. Cells with Nlk deletion or knock-in of the Raptor S863 phosphorylation mutants are defective in the rapid mTORC1 inhibition upon osmotic stress. Our study reveals a function of NLK in stress-induced mTORC1 modulation and the underlying biochemical mechanism of NLK in mTORC1 inhibition in stress response.

[Keywords: NLK; mTOR; Raptor; stress response; cancer]

Supplemental material is available for this article.

Received May 5, 2015; revised version accepted October 16, 2015.

The mechanistic target of rapamycin (mTOR; also known as mammalian TOR) is a highly conserved serine/threonine kinase. mTOR exists in two different complexes, mTORC1 and mTORC2, which are distinguished by their unique subunits, Raptor and Rictor, respectively (Laplante and Sabatini 2012). mTORC1 is a master regulator of cell growth and metabolism. Accordingly, it is modulated by a wide range of signals, including growth factors, nutrients, and stress (Sengupta et al. 2010). Growth factors and nutrients activate $\mathrm{mTORC} 1$ to promote cell growth by stimulating transcription, translation, and anabolism as well as inhibiting autophagy. Growth factors activate mTORC1 through the AKT-TSC1/TSC2-Rheb axis (Inoki et al. 2002), in which the Rheb GTPase binds to and activates mTORC1 (Inoki et al. 2003a; Saucedo et al. 2003; Stocker et al. 2003). Amino acids signal through the lysosomal Rag GTPases to stimulate mTORC1 (Kim et al. 2008; Sancak et al. 2008). Upon amino acid stimulation, the active Rag heterodimers physically bind to the Raptor subunit of mTORC1 and thus recruit mTORC1 to lyso-

Corresponding author: kuguan@ucsd.edu Article is online at http://www.genesdev.org/cgi/doi/10.1101/gad.265116. 115 . somes (Sancak et al. 2008), where it is activated by the lysosomally localized Rheb. The Rag GTPases are activated by amino acids via the Ragulator (Sancak et al. 2010; Bar-Peled et al. 2012) and GATOR1 (Bar-Peled et al. 2013) complex, which function as activator (GEF) and inhibitor (GAP) for Rag GTPases, respectively. Although Rag GTPases have been considered as the major factors mediating amino acid signaling to $\mathrm{mTORC1}$, glutamine, but not leucine, could activate mTORC1 through the Arf1 GTPase in cells when Rag GTPases are deleted, thus suggesting differential regulation of $\mathrm{mTORC} 1$ by distinct amino acids (Jewell et al. 2015).

As a central cell growth controller, mTORC1 is potently inhibited by stress conditions such as hypoxia, oxidative stress, and hyperosmotic stress. Under physiological condition, $\sim 1 \%-3 \%$ of the oxygen consumed by cells is metabolized to reactive oxygen species (ROS), which generates oxidative stress in the cell. Oxidative stress

(C) 2015 Yuan et al. This article is distributed exclusively by Cold Spring Harbor Laboratory Press for the first six months after the full-issue publication date (see http://genesdev.cshlp.org/site/misc/terms.xhtml). After six months, it is available under a Creative Commons License (Attribution-NonCommercial 4.0 International), as described at http:// creativecommons.org/licenses/by-nc/4.0/. 
potently and rapidly inhibits $\mathrm{mTORC1}$, possibly through activating AMPK kinase (Chen et al. 2010). Osmotic balance is another essential factor for cell survival and growth. In mammals, normal tonicity in most tissues is $300 \mathrm{mOsm} / \mathrm{kg}$. However, in renal medulla, local tonicity can reach as high as 600-1700 mOsm $/ \mathrm{kg}$. Hyperosmotic stress rapidly inhibits mTORC1. One possible mechanism is through activating a calyculin A-sensitive phosphatase (Parrott and Templeton 1999). Hyperosmotic stress may also inhibit mTORC1 activity through its upstream regulator (Akt) (Chen et al. 1999) or AMPK (Barnes et al. 2002; Hawley et al. 2010). It has been suggested that the low mTOR activity retained under moderate hypertonic conditions facilitates the expression of some osmoresponsive genes (Ortells et al. 2012). A previous study has implicated JNK in osmotic stress-induced mTORC1 regulation. Perplexingly, JNK was reported to enhance mTORC1 activity in response to osmotic stress, although hyperosmotic conditions strongly inhibited the phosphorylation of mTORC1 substrates (Kwak et al. 2012). It is thus not clear how mTORC1 is regulated under hyperosmotic condition if JNK activates $\mathrm{mTORC1}$ and mediates the osmotic signal.

The Nemo-like kinase (NLK) was originally identified as a regulator in Drosophila photoreceptor development (Choi and Benzer 1994). NLK is a distinct member of the MAP kinase (MAPK) subfamily. It is notable that the activation loop of NLK protein possesses the sequence Thr-Gln-Glu (TQE) motif instead of a TXY motif that is usually seen in other MAPK members (Brott et al. 1998). Glutamate (E), a negatively charged amino acid, can mimic the effect of phosphorylation. A recent study showed that NLK could be activated by homodimerization and autophosphorylation on the Thr residue in the TQE motif (Ishitani et al. 2011). Thus, NLK can be activated without being phosphorylated in the activation loop by upstream kinases. Moreover, high levels of ectopic expression could also result in artificial NLK activation. Like other members of MAPK family, NLK is a proline-directed kinase that preferentially phosphorylates a Ser-Pro or Thr-Pro motif. The best-known function of NLK is in the regulation of Wnt signaling by phosphorylating the mammalian T-cell factor (TCF)/lymphoid enhancer factor (LEF) (Ishitani et al. 1999, 2003b; Thorpe and Moon 2004; Ota et al. 2012). NLK is also implicated in mediating signaling events such as IL-6 (Kojima et al. 2005), TGF- $\beta$ (Ohkawara et al. 2004; Kojima et al. 2005), NGF (Ishitani et al. 2009), and Notch1 (Ishitani et al. 2010).

In a screen for new regulators of the MTORC1 pathway, we identified NLK as a negative modulator of mTORC1 signaling. We found that NLK is activated by stress conditions such as hyperosmotic stress and oxidative stress. Sequence analysis shows that NLK is most closely similar to the Hog 1 protein in the yeast proteome. Hog1 is also a member of the MAPK family and is the major osmoresponsive regulator in yeast (Hohmann et al. 2007). Hog1 is highly homologous to the mammalian p38 MAPK (Han et al. 1994), which is one of the major stress-responsive kinases in mammalian cells (Han et al. 1994; Whitmarsh 2010). This study reveals the cellular function and molecular mechanism of NLK in stress-induced mTORC1 inhibition.

\section{Results \\ NLK mediates stress-induced mTORC1 inhibition}

We screened a human kinome library to search for new mTORC1 regulators. An individual kinase was cotransfected with HA-S6K1, and phosphorylation of the mTORC1 site in S6K1 was detected by p-S6K antibody (Supplemental Fig. S1A). We found that overexpression of NLK, but not its kinase-negative (KN) mutant, significantly inhibited mTORC1, as indicated by the decreased S6K1 and 4EBP1 phosphorylation (Fig. 1A; Supplemental Fig. S1B,C). NLK did not affect Akt phosphorylation on S473, the target of mTORC2 complex (Fig. 1A; Supplemental Fig. S1D; Sarbassov et al. 2005), suggesting the specificity of NLK on mTORC1 inhibition. Previous studies have suggested that TAK1 is an upstream activating kinase of NLK (Ishitani et al. 1999, 2003a; Kanei-Ishii et al. 2004; Ohkawara et al. 2004; Smit et al. 2004). However, in our experiments, overexpression of TAK1 had no effect on mTORC1 signaling (Supplemental Fig. S1E).

mTORC1 activation requires localization on lysosomes. We next examined whether mTOR localization was affected by NLK. As shown in Figure 1, B and C, when NLK-expressing or NLK-nonexpressing cells were compared in coculture, the lysosomal localization of mTOR, as indicated by the lysosome marker LAMP2, was significantly reduced in the NLK-expressing cells. In contrast, expression of the kinase-inactive NLK-KN had no effect on mTOR localization. The above results suggest that NLK inhibits mTORC1, possibly by interfering with mTOR lysosome localization.

mTORC1 is inhibited by various stress conditions, including amino acid starvation, glucose starvation, hyperosmolarity, and oxidative stress (Supplemental Fig. S1G). Our previous studies have shown that amino acids and glucose act through the Rag GTPase and AMPK, respectively, to regulate mTORC1 (Inoki et al. 2003b; Kim et al. 2008). In this study, we focused on stress signals to mTORC1 inhibition. We further found that hyperosmotic stress (treatment with sorbitol or $\mathrm{NaCl}$ ) and oxidative stress (treatment with $\mathrm{H}_{2} \mathrm{O}_{2}$ or menadione) rapidly inhibited mTORC1 in HCT116, HeLa, and NIH3T3 cells, except that menadione did not inhibit mTORC1 in HeLa cells (Supplemental Fig. S2), indicating cell type-independent mTORC1 inhibition by these stress conditions.

To investigate the biological relevance of NLK in mTORC1 regulation, we determined NLK activation in response to multiple conditions that are known to inhibit mTORC1. Hyperosmotic stress, oxidative stress, and amino acid starvation all potently inhibit mTORC1. Because high levels of NLK expression lead to autoactivation, we characterized stable lines with low levels of NLK expression and low basal kinase activity. Notably, NLK was activated by hyperosmotic stress induced by sorbitol and oxidative stress induced by $\mathrm{H}_{2} \mathrm{O}_{2}$, but not amino acid starvation, in both HEK293 cells (Fig. 1D) and mouse 
A

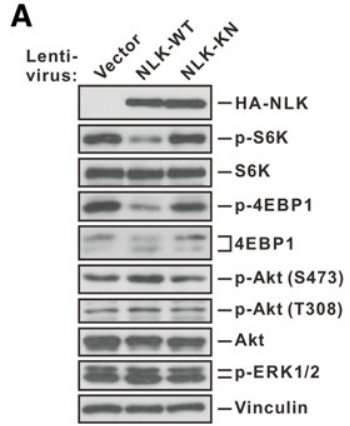

C
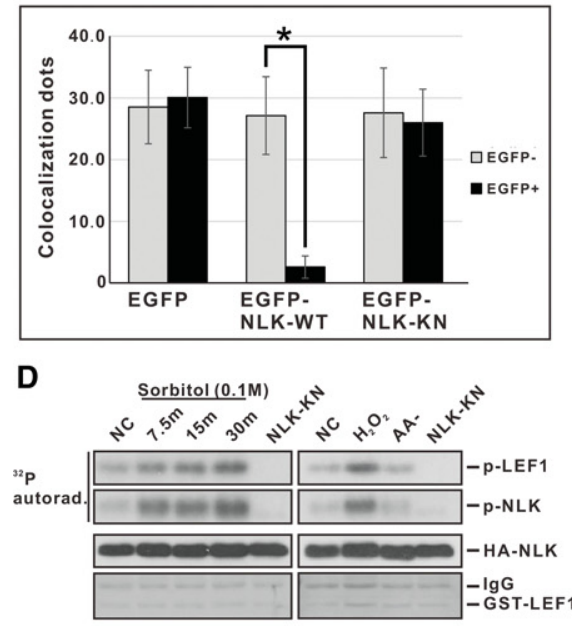

$\mathbf{F}$

Time: $\mathrm{NC} 3 \mathrm{~min} .5 \mathrm{~min} 15 \mathrm{~min} 30 \mathrm{~min} 60 \mathrm{~min}$

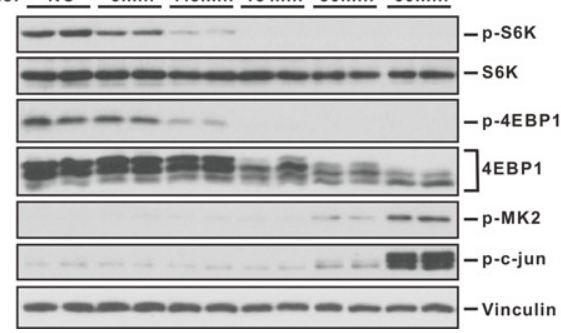

B
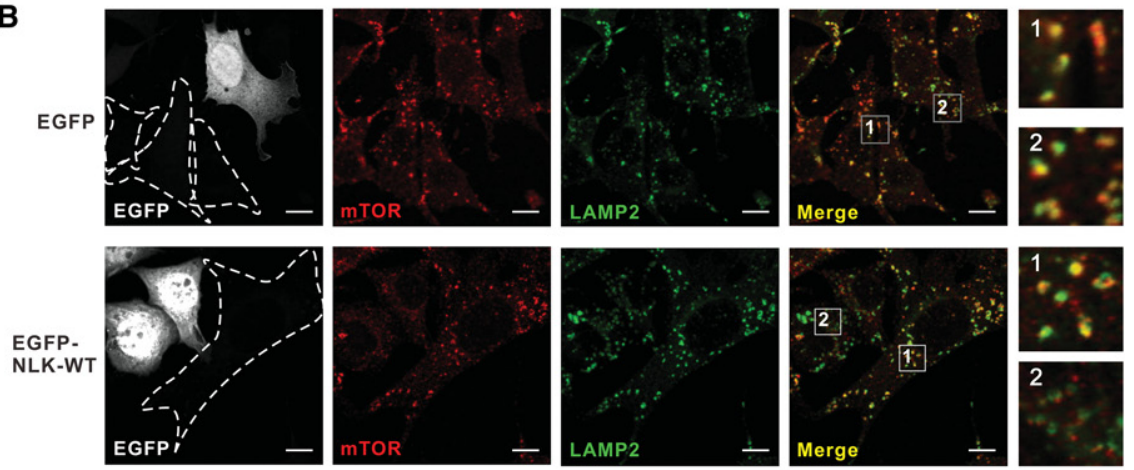

$2=$
4
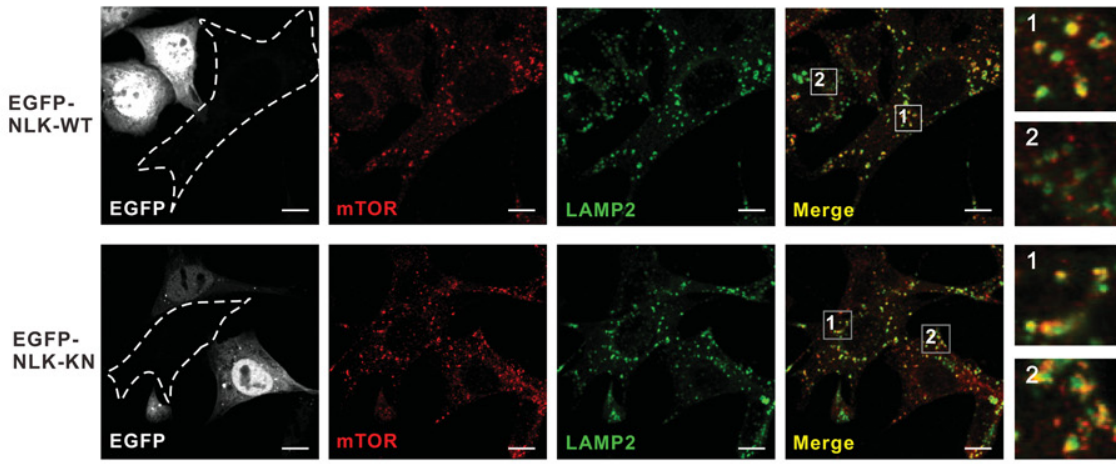

E MTOR
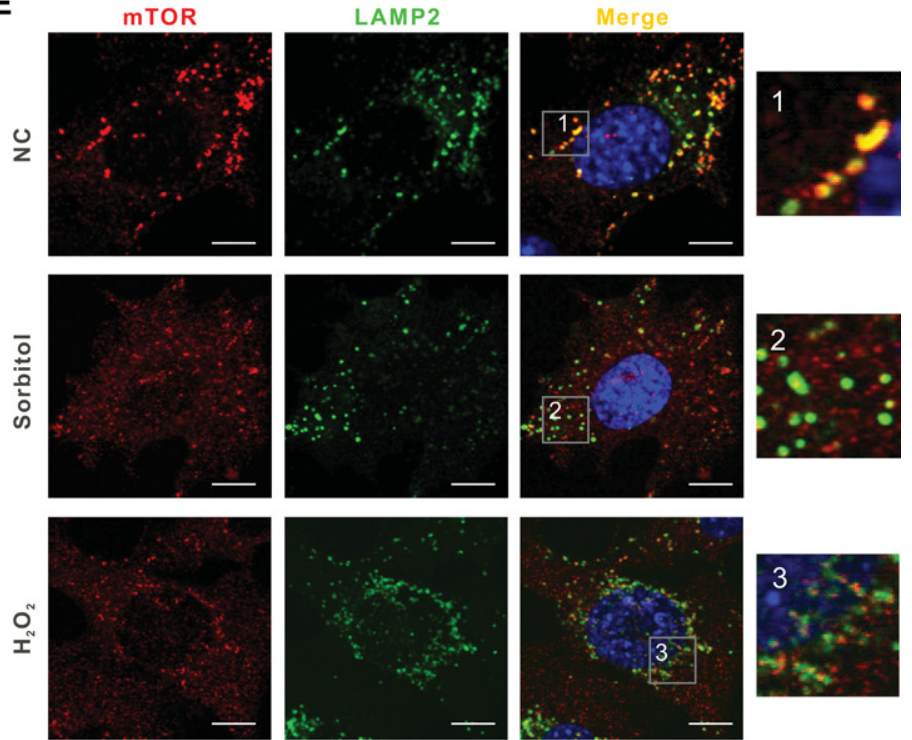

Figure 1. NLK inhibits mTORC1. (A) mTORC1 is inhibited by NLK overexpression. Cells were infected with lentivirus expressing empty vector, NLK wild type (NLK-WT), or NLK-KN. Phosphorylated or total amount of proteins were detected with corresponding antibodies as indicated. (B) mTOR localization on lysosomes is impaired by NLK. EGFP-tagged NLK wild type or NLK-KN was transfected by electroporation into mouse embryonic fibroblast (MEF) cells. Endogenous mTOR and LAMP2 were immunostained with specific antibodies. NLK expression was demonstrated by EGFP signal. In the field, the EGFP-NLK transfected cells were visualized by EGFP, while the untransfected cells were circled by dashed lines. The right panels with numbers are magnified from either EGFP-negative (1) or EGFP-positive (2) cells. Inhibition of mTORC1 by EGFP-NLK was confirmed by Western blot (Supplemental Fig. S1F). Bars, $10 \mu \mathrm{m}$. (C) Quantification of mTOR and LAMP2 colocalization. Fifteen to 20 cells with or without EGFP signal were randomly selected and counted. An asterisk indicates a statistically significant difference $(P<0.05)$. Statistical analysis is described in the Materials and Methods. $(D)$ NLK is activated by stress conditions in HEK293 cells. HEK293 cells stably expressing HA-tagged NLK were treated with $0.1 \mathrm{M}$ sorbitol for the indicated times, $0.25 \mathrm{mM} \mathrm{H}_{2} \mathrm{O}_{2}$ for $30 \mathrm{~min}, 2 \mathrm{mM}$ DTT for $1 \mathrm{~h}$, or amino acid starvation for $1 \mathrm{~h}$. HA-NLK was immunoprecipitated from cell lysates and subjected to in vitro kinase assay using purified GST-LEF1-51-210 as substrate. Phosphorylation of LEF1 and autophosphorylation of NLK were detected by ${ }^{32} \mathrm{P}$-autoradiography. The bottom panel shows Commassie Brilliant Blue staining of the membrane. Cells stably expressing NLK-KN were used as a control. (E) Hyperosmotic stress disrupts mTORC1 lysosomal localization. MEF cells were treated with $0.2 \mathrm{M}$ sorbitol for $15 \mathrm{~min}$ followed by immunostaining with mTOR and LAMP2 antibody. Magnified view fields are shown at the right. Bars, $10 \mu \mathrm{m}$. (F) mTORC1 inhibition occurs prior to the activation of $\mathrm{p} 38$ or JNK upon hyperosmotic stress. HEK293 cells were treated with $0.2 \mathrm{M}$ sorbitol for the times indicated. Phosphorylation of S6K, 4EBP1, MK2 (p38 substrate), and c-jun (JNK substrate) was detected with specific antibodies. See also Supplemental Figures S1 and S2. 
embryonic fibroblasts (MEFs) (Supplemental Fig. S1H). Our observations suggest that NLK may selectively mediate stress-induced mTORC1 inhibition. To further test this possibility, we analyzed mTORC1 localization upon stress conditions. Similar to the results from NLK overexpression, hyperosmotic or oxidative stress impaired mTOR colocalization with lysosomes (Fig. 1E; Supplemental Fig. S1I). We did observe a small fraction of mTOR that was still colocalized with lysosomes, probably because of the short treatment time ( $15 \mathrm{~min})$. Notably, although it did not condense in large speckles as lysosomes, mTOR still existed in smaller granules, which were likely stress granules as described previously (Takahara and Maeda 2012; Thedieck et al. 2013; Wippich et al. 2013).

Since p38 and JNK MAPK are two major regulators in hyperosmotic stress (Han et al. 1994; Whitmarsh 2010), we investigated whether they might be involved in stress-induced mTORC1 inhibition. Phosphorylation of MK2 and c-Jun was used as an indicator for activation of p38 and JNK, respectively. A time course of sorbitol treatment clearly showed that mTORC1 inhibition $(7.5 \mathrm{~min})$ occurred much earlier than a significant activation of p38 or JNK (60 min) (Fig. 1F), thus arguing that mTORC1 inhibition by hyperosmotic stress is not mediated by $\mathrm{p} 38$ or JNK. Consistently, treatment of cells with p38 or JNK inhibitors did not affect mTOR inhibition upon hyperosmotic stress (Supplemental Fig. S1J). Furthermore, amino acid-stimulated or glucose-stimulated mTORC1 reactivation was blocked in the presence of hyperosmotic stress (Supplemental Fig. S1K), suggesting that hyperosmotic stress inhibits $\mathrm{mTORC} 1$ at a node downstream from amino acid and glucose signaling.

\section{Loss of NLK compromises stress-induced mTORC1 inhibition and impairs physiological response to stress}

To confirm that NLK is involved in stress-induced mTORC1 inhibition, we generated Nlk knockout cells using the CRISPR/Cas9 genome-editing system. Two different Nlk knockout lines were generated with different guide sequences (Supplemental Fig. S3A-C). The Nlk knockout cells showed normal mTORC1 activity under normal culture condition (Fig. 2A). However, both Nlk knockout lines showed resistance to mildly hyperosmotic stress-induced mTORC1 inhibition within $30 \mathrm{~min}$, as monitored by the phosphorylation of S6K and 4EBP1 (Fig. 2A; Supplemental Fig. S4A). Similar results were

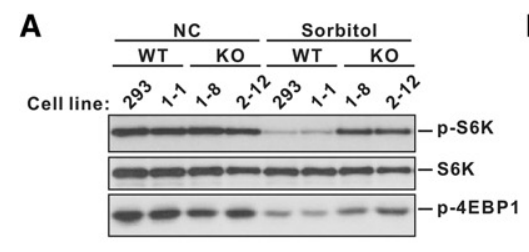

D
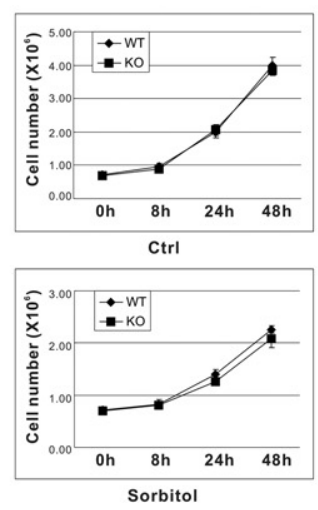

B

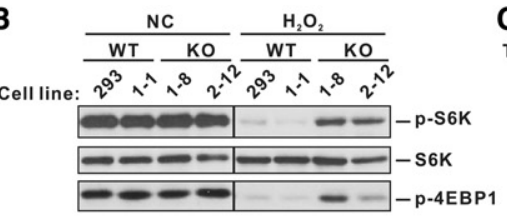

E

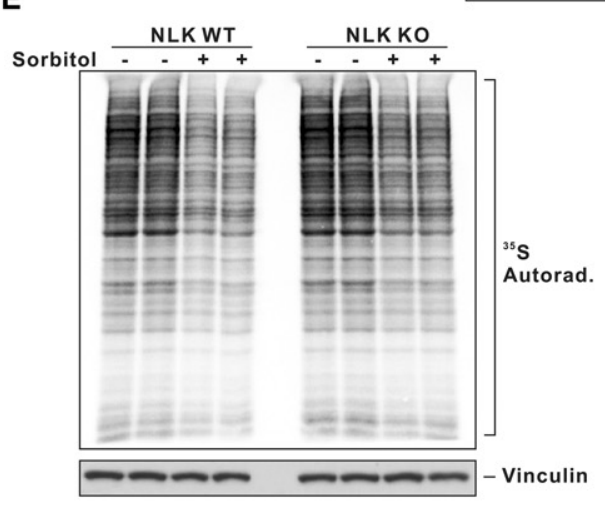

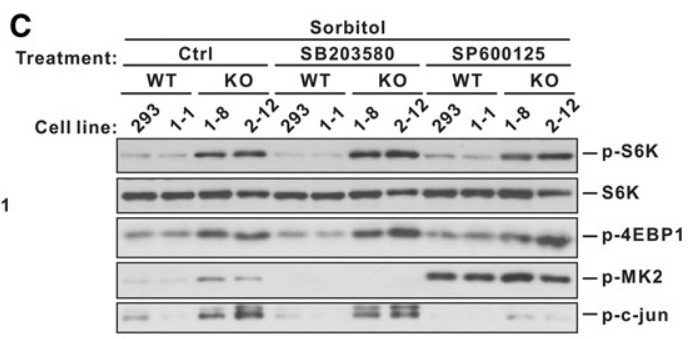

$\mathbf{F}$

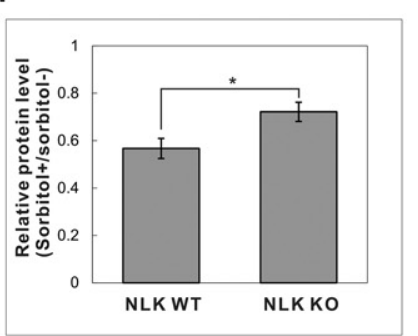

Figure 2. Loss of NLK compromises stress-induced mTORC1 inhibition and physiological response. $(A)$ NIk deletion compromises mTORC1 inhibition upon mild hyperosmotic stress. Wild-type (293 and 1-1 clone) or Nlk knockout (1-8 and 2-12 clone) cells were cultured in normal conditions (NC) or treated with $0.1 \mathrm{M}$ sorbitol for $10 \mathrm{~min}$. Phosphorylation of S6K and 4EBP1 was detected with specific antibodies. (B) NLK deficiency compromises oxidative stress-induced mTORC1 inhibition. Wild-type or Nlk knockout cells were in normal culture (NC) or treated with $0.25 \mathrm{mM} \mathrm{H}_{2} \mathrm{O}_{2}$ for $15 \mathrm{~min}$. Phosphorylation of S6K and 4EBP1 was detected with specific antibodies. (C) NLK signaling to mTORC1 is independent of p38 and JNK MAPK. Wild-type or Nlk knockout cells were pretreated with DMSO, $10 \mu \mathrm{M}$ SB203580, or $20 \mu \mathrm{M}$ SP600125 for $30 \mathrm{~min}$. Cells were then kept in normal culture (NC) or treated with $0.1 \mathrm{M}$ sorbitol for $10 \mathrm{~min}$ in the presence of inhibitors. Activity of mTORC1, p38, and JNK was detected with specific antibodies. $(D)$ Nlk deletion synergizes with inhibition of p38 and JNK to suppress cell growth under hyperosmotic condition. The data represent results from three independent experiments. (E) NLK deficiency compromises translation inhibition in response to hyperosmotic stress. ${ }^{35} \mathrm{~S}$ labeling of newly synthesized protein was detected by autoradiography. $(F)$ Quantification of the results in E. Experiments were repeated three times. Data shown are relative protein synthesis normalized to the control without sorbitol treatment (see also Supplemental Figs. S3, S4). 
observed when cells were treated with hyperosmotic stress induced by $\mathrm{NaCl}$ (Supplemental Fig. S4B). Inhibition of mTORC1 by $\mathrm{H}_{2} \mathrm{O}_{2}$-induced oxidative stress was also compromised in the Nlk knockout cells, although to a lesser degree (Fig. 2B). To further confirm the biological effect of NIk deletion, we knocked out the Nlk gene in Neuro-2a cells and observed that Nlk knockout cells also exhibited resistance to hyperosmotic stress-induced or oxidative stress-induced mTORC1 inhibition (Supplemental Fig. S4C,D). These results suggest that NLK plays a role in stress-induced mTORC1 inhibition in a cell typeindependent manner.

Phosphatase activation by hyperosmotic stress has been suggested to be responsible for S6K suppression (Parrott and Templeton 1999; Kwak et al. 2012). However, the time course of S6K dephosphorylation by Torin1, a specific inhibitor of mTOR, was similar between wild-type and Nlk knockout cells (Supplemental Fig. S4E), suggesting that S6K phosphatase activity was not altered in the NIk knockout cell. Our data indicate that loss of NLK may directly affect mTORC1 regulation by osmotic stress. In addition, treatment of cells with p38 inhibitor (SB203580) or JNK inhibitor (SP600125 or JNK-IN-8) did not affect $\mathrm{mTORC1}$ response to mild hyperosmotic stress in either wild-type or N1k knockout cells (Fig. 2C; Supplemental Fig. S4F), consistent with the notion that p38 and JNK are not involved in the osmotic stress-induced mTORC1 inhibition. Interestingly, we noted that p38 and JNK activity showed a compensatory increase in NIk knockout cells upon stress. Since p38 and JNK are both MAPKs involved in stress response, the up-regulation of p38 and JNK indicates a compensatory response in cells when Nlk is deleted. These observations are consistent with the notion that NLK may function in parallel to $\mathrm{p} 38$ and JNK in mediating stress responses.

We next tested the physiological outcomes of Nlk deletion. Upon mild osmotic stress, cell growth and the majority of anabolic processes are suppressed, while osmoprotective genes are induced (Burg et al. 2007). Cells are able to adapt to a hypertonic environment within $20 \mathrm{~h}$ and resume normal growth and functions (Burg et al. 2007). As expected, sorbitol reduced HEK293 cell growth (Fig. 2D). Surprisingly, Nlk knockout cells showed only little difference in cell growth when compared with wild-type cells. We reasoned this could be due to the compensatory activation of p38 or JNK in the N1k knockout cells, as discussed above (Fig. 2C), which may protect cells from hyperosmotic stress. Therefore, we treated wild-type and Nlk knockout cells with inhibitors of p38 and JNK. Notably, treatment with a low concentration of p38 and JNK inhibitors preferentially inhibited the growth of Nlk knockout cells over the wild-type cells (Fig. 2D). This result suggests that NLK acts together with p38 and JNK in the osmotic stress response. The immediate shutdown of mTORC1 signaling mediated by NLK does play a role in cell adaptation to mildly hypertonic condition. It is conceivable that the ineffectiveness of mTORC1 inhibition in the Nlk knockout cells by osmotic stress may fail to suppress consumption of building materials and energy that are essential for synthesis of osmoprotec- tive factors. Consistent with a role of mTORC1 in translation regulation, we observed that sorbitol-induced translation inhibition was significantly compromised in the N1k knockout cells (Fig. 2E,F). Collectively, our results demonstrate a role of NLK in cellular osmotic stress response.

\section{NLK inhibits mTORC1 through Rag GTPases-dependent signaling}

We investigated the mechanism of NLK in inhibiting mTORC1 signaling. Amino acids potently stimulate mTORC1 by promoting lysosomal translocation (Sancak et al. 2008). We found that amino acid-induced mTORC1 activation was inhibited by NLK overexpression (Fig. 3A). The Rag GTPases play a key role in amino acid signaling to mTORC1. Overexpression of the constitutively active Rag complex (RagA-Q66L/RagC-S75N) activated mTORC1 even under amino acid starvation (Fig. 3B). Interestingly, NLK could suppress the Rag-induced mTORC1 activation (Fig. 3B), suggesting that NLK may act between Rag GTPases and mTORC1. It has been well demonstrated that amino acids are required for full mTORC1 activation by insulin and serum (Kim et al. 2008; Sancak et al. 2008). If NLK blocks Rag signaling to mTORC1, then it should also suppress mTORC1 activation by insulin and serum. Overexpression of NLK indeed reduced mTORC1 activation by either insulin or FBS (Supplemental Fig. S5A).

To further test the relationship between Rag and NLK, we examined the $\operatorname{Rag} A$ knockout HEK293A cells, which have reduced but still substantial $\mathrm{mTORC} 1$ activity due to Rag-independent mTORC1 activation (Jewell et al. 2015). Empty vector or NLK was transfected together with S6K into wild-type and RagA knockout cells. Notably, NLK expression dramatically decreased S6K phosphorylation in wild-type cells but not in $\operatorname{RagA}$ knockout cells (Fig. 3C). Consistently, hyperosmotic stress could not effectively inhibit mTORC1 in the $\operatorname{RagA}$ knockout cells (Fig. 3D). However, the S6K phosphorylation in the $\operatorname{Rag} A$ knockout cells was dependent on mTORC1, as rapamycin treatment inhibited S6K phosphorylation (Fig. 3C). The above results show that RagA is required for NLK to inhibit mTORC1 and further suggest that NLK may inhibit mTORC1 through the Rag-dependent pathway.

Considering the fact that Rag GTPases recruit mTORC1 to lysosome and NLK blocks mTOR lysosomal localization (Fig. 1B), we proposed the following two possibilities: (1) NLK inhibits mTORC1 through disrupting lysosomal localization of the Rag complex, or (2) NLK impairs the interaction between Rag and mTORC1. We tested the first possibility by checking Rag localization with NLK overexpression. Unlike mTORC1, Rag localization on lysosomes was not affected by NLK overexpression (Fig. 3E,F), thus arguing against a role of NLK in interfering Rag lysosomal localization. It has been reported that the constitutively active Rag complex cannot rescue osmotic stress-induced mTORC1 inhibition (Kim et al. 2008). Consistently, we found that, in the cells that express the constitutively active Rag complex, hyperosmotic stress or oxidative stress still impaired mTOR localization on the lysosomes 
A

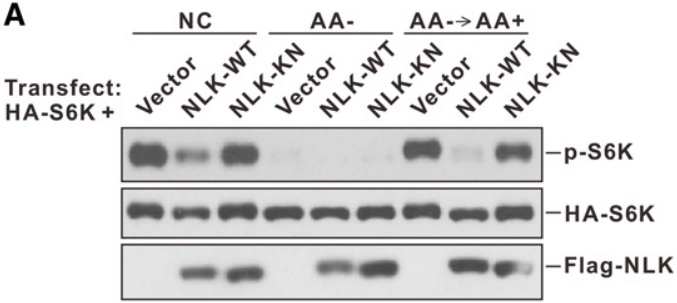

B

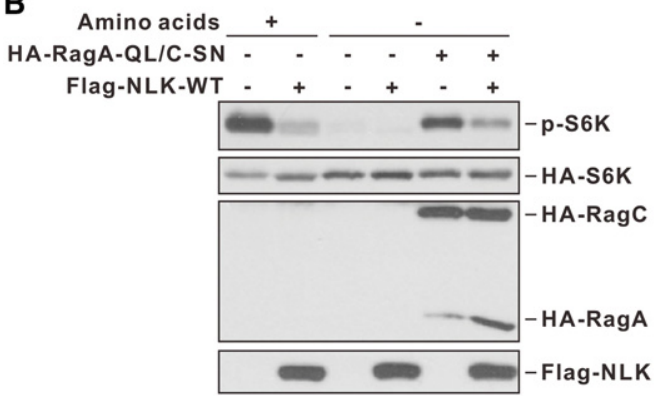

C

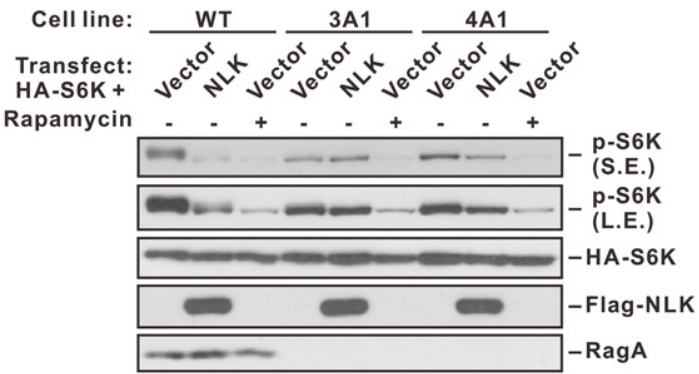

D

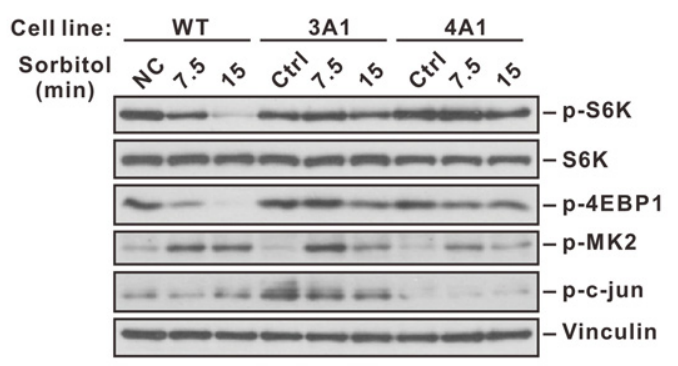

E
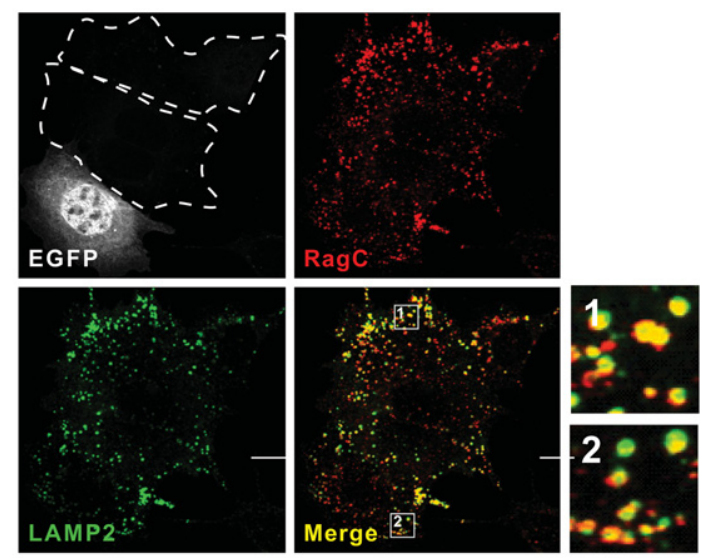

$\mathbf{F}$

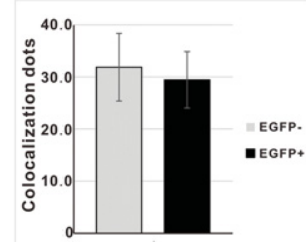

Figure 3. NLK inhibits mTORC1 through Rag GTPase-dependent signaling. (A)NLK dampens mTORC1 activation by amino acids. Empty vector, NLK wild type (NLK-WT), or NLK-KN was transfected together with S6K into HEK293 cells. Cells were starved with amino acid-free medium for $1 \mathrm{~h}$ and stimulated with amino acids for $15 \mathrm{~min}$. mTORC1 activity was examined by S6K phosphorylation. $(B)$ NLK blocks amino acid-stimulated or Rag-stimulated mTORC1 activation. Empty vector, NLK wild type, or NLK-KN was transfected together with S6K into HEK293 cells. Cells were starved with amino acid-free medium for $1 \mathrm{~h}$ and replenished with amino acids for $15 \mathrm{~min}$. HA-RagA-QL/C-SN denotes the cotransfection of the constitutively active Rag GTPase complex, which activates mTORC1 in the absence of amino acids. Activity of mTORC1 was examined by S6K phosphorylation. (C) RagA is required for NLK to inhibit mTORC1. Empty vector or NLK wild type and S6K were cotransfected into wild-type or RagA knockout HEK293A cells (clones 3A1 and 4A1). Rapamycin (20 nM) was included as a positive control to inhibit mTORC1 activity. (S.E.) Short exposure; (L.E.) long exposure. (D) RagA deficiency compromises mTORC1 inhibition by hyperosmotic stress. Wild-type or RagA knockout cells were in normal culture (NC) or treated with $0.1 \mathrm{M}$ sorbitol for the times indicated. Phosphorylation of S6K, 4EBP1, and p38 and JNK substrates was detected with specific antibodies. $(E)$ RagC localization on lysosomes is not affected by NLK. EGFP-tagged NLK wild-type was transfected by electroporation into MEF cells. Endogenous RagC and LAMP2 were immunostained with specific antibodies. NLK expression was demonstrated by EGFP signal. In the field, the EGFP-NLK transfected cells were visualized by EGFP; the untransfected cells are circled by dashed lines. The right panels with numbers were magnified from either EGFP-negative (1) or EGFP-positive (2) cells. Bars, $10 \mu \mathrm{m}$. (F) Quantification of mTOR dots that show colocalization with lysosome marker LAMP2. Fifteen to 20 cells with or without EGFP signal were randomly selected and counted (see also Supplemental Fig. S5).

(Supplemental Fig. S5B,C). These results indicate that stress may disrupt the interaction between Rag and mTORC1.

\section{NLK inhibits mTORC1 by disrupting Raptor-Rag interaction}

We examined the interaction between the Rag complex and Raptor, which is the mTORC1 subunit directly inter- acting with Rag, and found that the interaction between Raptor and the Rag complex (either the wild-type or constitutively active form) was reduced by NLK (Fig. 4A). It is of interest that an upshift band of Raptor appeared when NLK was cotransfected. This upshift of Raptor is likely due to NLK-induced phosphorylation because $\lambda$ phosphatase treatment abolished the upshift band of Raptor (Fig. 4B). Reloading of cell lysate and immunoprecipitation samples together clearly showed that the Raptor protein 
Yuan et al.
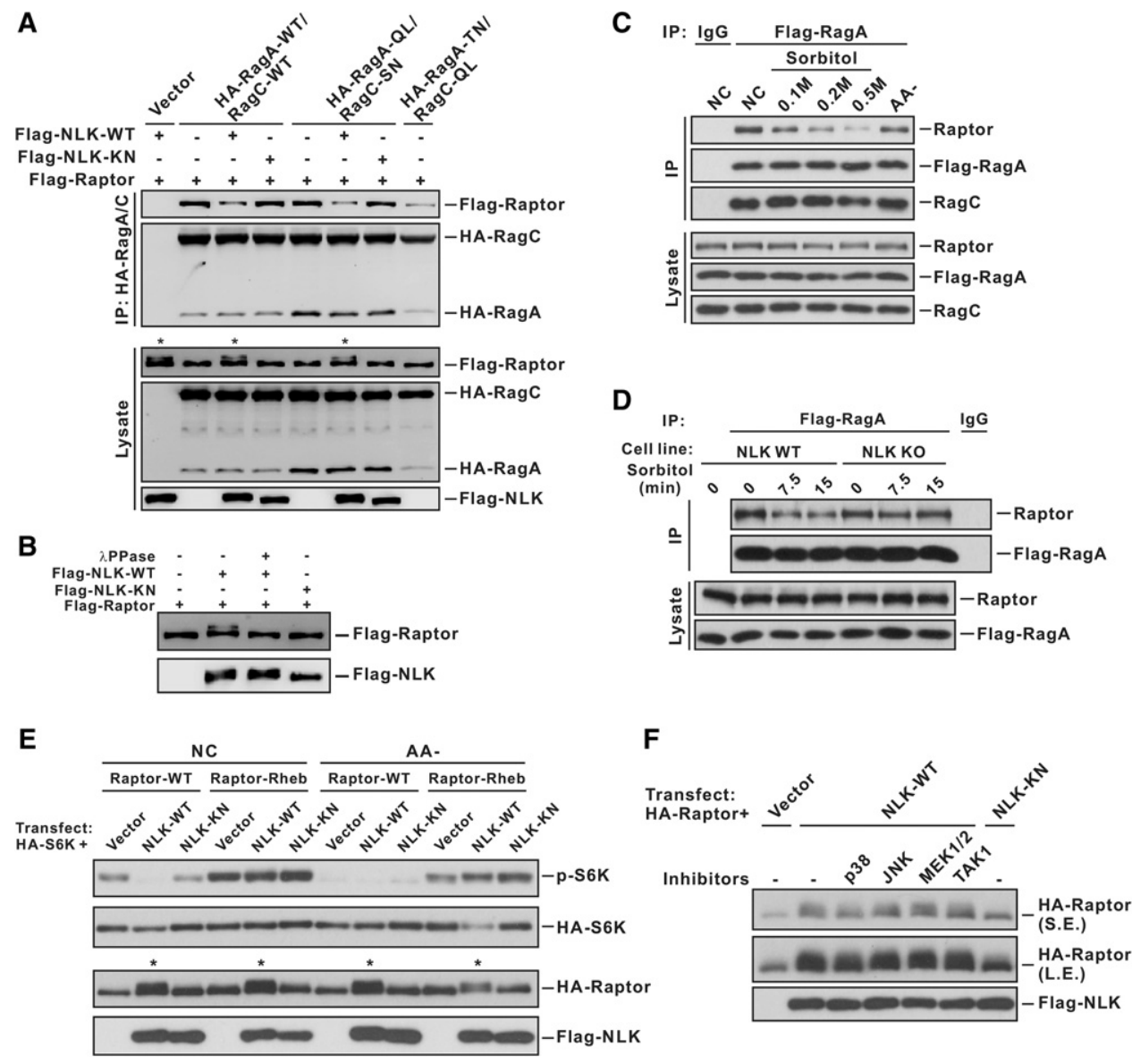

Figure 4. NLK inhibits mTORC1 by disrupting Raptor-Rag interaction. (A) NLK disrupts Raptor-Rag interaction. Empty vector, NLK wild type (NLK-WT), or NKL-KN was transfected together with Raptor and wild-type, constitutively active (RagA-QL/RagC-SN), or dominant-negative (RagA-TN/RagC-QL) Rag complexes. The interactions between Raptor and Rag complexes were examined by coimmunoprecipitation (co-IP). Asterisks denote lanes where Raptor shows an upshift due to NLK cotransfection. $(B)$ NLK induces Raptor phosphorylation. Empty vector, NLK wild type, or NLK-KN was transfected together with Raptor. Cell lysate of the NLK wild-type sample was incubated with or without $\lambda$ phosphatase ( $\lambda$ PPase) as indicated. Raptor mobility was examined by Western blot. (C) Hyperosmotic stress disrupts Raptor-Rag interaction. HEK293T cells stably expressing Flag-tagged RagA were treated with sorbitol at different concentrations for 15 min or starved with amino acid-free medium $\left(\mathrm{AA}^{-}\right)$for $1 \mathrm{~h}$. The interaction between endogenous Raptor and transfected Rag complex was examined by co-IP with Flag-RagA. $(D)$ NLK is required for hyperosmotic stress to disrupt Raptor-Rag interaction. Wild-type or Nlk knockout cells stably expressing Flag-tagged RagA were treated with $0.2 \mathrm{M}$ sorbitol for the indicated times. The interaction between endogenous Raptor and ectopic RagA was examined by co-IP with Flag-RagA. (E) Anchoring Raptor on lysosomes makes mTORC1 resistant to inhibition by NLK. Empty vector, NLK wild type, or NLK-KN was transfected into HEK293 cells together with S6K and wildtype Raptor or Raptor fused with C-terminal 15 amino acid residues of Rheb (Raptor-Rheb). Cells were in normal culture or starved with amino acid-free medium for $1 \mathrm{~h}$. The activity of mTORC1 was examined by S6K phosphorylation. (F) NLK induces Raptor mobility shift. Empty vector, NLK wild type, or NLK-KN was transfected into HEK293 cells together with Raptor. NLK wild-type-expressing cells were in normal culture or treated with $10 \mu \mathrm{M}$ p38, $20 \mu \mathrm{M}$ JNK, $10 \mu \mathrm{M}$ MEK1/2, or $0.3 \mu \mathrm{M}$ TAK1 inhibitors for 30 min before harvest. The mobility of Raptor was examined by SDS-PAGE (see also Supplemental Fig. S6).

coprecipitated with Rag contained little of the upshifted species (Supplemental Fig. S6A), indicating that only the unphosphorylated Raptor was in complex with Rag. We further tested the effect of osmotic stress and oxidative stress on the Raptor-Rag interaction in an HEK293 cell line stably expressing Flag-RagA. Hyperosmotic stress or oxidative stress treatment reduced association of Rag with endogenous Raptor (Fig. 4C; Supplemental Fig. S6B). Importantly, hyperosmotic stress failed to disrupt Rag-Raptor interaction in Nlk knockout cells (Fig. 4D). These data are consistent with a model in which NLK me- diates the osmotic stress and oxidative stress signals to inhibit Rag-Raptor interaction.

If the main mechanism of NLK to inhibit mTORC1 is to block Rag-Raptor interaction and mTORC1 lysosomal localization, then artificial targeting of Raptor to lysosomes would make mTORC1 resistant to NLK inhibition. To test this model, we used a Raptor construct (RaptorRheb) that had fusion of the Rheb C-terminal 15 amino acid residues, which constitute the lysosomal localization signal (Sancak et al. 2010). Expression of Raptor-Rheb rendered S6K resistant to amino acid starvation as well as 
sorbitol treatment (Fig. 4E; Supplemental Fig. S6C). Moreover, NLK inhibition on S6K was also abolished by Raptor-Rheb. Notably, NLK expression still induced an upshift of Raptor-Rheb fusion (Fig. 4E), indicating a decoupling between the phosphorylation of Raptor and mTORC1 inhibition in the Raptor-Rheb-expressing cells. These results show that constitutive lysosomal localization of mTORC1 could escape from inhibition by osmotic stress and NLK and that NLK does not directly inhibit mTORC1 activity. Treatment with various inhibitors showed that the NLK-induced Raptor mobility shift was not affected by inhibition of p38, JNK, MEK1/2, or TAK1 (Fig. 4F), again suggesting that NLK functions in a mechanism independent of these kinases.

\section{NLK is required for stress-induced Raptor phosphorylation}

NLK is a MAPK family member and phosphorylates the Thr-Pro or Ser-Pro motif. We used an antibody recognizing the p-Thr-Pro motif to characterize the phosphorylation of Raptor by NLK. This antibody, according to the manufacturer's instruction, may also detect the p-SerPro motif. Overexpression of NLK, but not its KN mutant, led to a strong phosphorylation signal of Raptor, as detected by the p-Thr-Pro antibody (Fig. 5A). To study the relationship of Raptor phosphorylation and its association with the Rag complex, we performed a sequential immunoprecipitation as described in the legend for Figure 5B. The results showed that the phosphorylated Raptor did not interact with the Rag complex and remained in the supernatant after Rag immunoprecipitation (Fig. 5B). In contrast, the unphosphorylated Raptor was found in the Rag immunoprecipitation. These data are consistent with a model in which NLK disrupts Raptor-Rag association through inducing Raptor phosphorylation.

We examined endogenous Raptor phosphorylation in response to stress. Hyperosmotic stress, but not amino acid starvation, rapidly induced Raptor phosphorylation at the p-Thr-Pro (or p-Ser-Pro) motif (Fig. 5C; Supplemental Fig. S6D). Importantly, hyperosmotic stress failed to induce Raptor phosphorylation in N1k knockout cells (Fig. 5D), demonstrating the essential in vivo role of NLK in Raptor phosphorylation upon osmotic stress. Moreover, oxidative stress also induced Raptor phosphorylation (Fig. 5D).

\section{NLK phosphorylates Raptor at residue $S 863$}

A straightforward model for the above observation is that NLK directly phosphorylates Raptor at the Thr-Pro or Ser-Pro site. We examined in vitro phosphorylation of immunopurified mTORC1 complex and found that Raptor, but not other subunits of the mTORC1 complex, was significantly phosphorylated by NLK in vitro (Supplemental Fig. S7A). To search for the phosphorylation sites on Raptor, different Raptor fragments were made and used as substrates for the in vitro kinase assay with purified NLK. The fragments containing residues 741-912 and 741-1000 showed strong phosphorylation signals (Supplemental Fig. S7B). We further narrowed down the region and mutated threonine and serine residues in the ThrPro or Ser-Pro motif. Interestingly, mutation of S877 to alanine significantly decreased phosphorylation of the 741912 fragment, while additional mutation of S863A largely abolished the phosphorylation by NLK (Fig. 5E).

To confirm whether S863 and S877 are involved in NLK regulation on $\mathrm{mTORC1}$, we tested the interaction between Rag and various Raptor mutants. Interestingly, Raptor mutant S863D, but not S877D, dramatically reduced its association with Rag (Fig. 5F). Moreover, the NLK-induced upshift of Raptor was abolished in the Raptor S863D mutant (Fig. 5F). Furthermore, the binding of Raptor S863A mutant to the Rag complex was not effectively inhibited by NLK when compared with the Raptor wild type (Fig. 5G). It is worth noting that S863 in Raptor is conserved from Drosophila to mammals (Supplemental Fig. S7C,D). These data suggest that Raptor S863 is a major NLK phosphorylation site, and its phosphorylation plays a key role in modulating the Raptor-Rag interaction.

\section{NLK inhibits mTORC1 by phosphorylating Raptor S863 residue}

To investigate the physiological significance of NLK phosphorylation on the Raptor S863 residue, we used an antibody specifically recognizing phospho-Raptor-S863 to examine endogenous Raptor phosphorylation. Similar to the results obtained with the p-Thr-Pro antibody (Fig. $5 \mathrm{C}, \mathrm{D})$, both hyperosmotic stress and oxidative stress induced Raptor-S863 phosphorylation in wild-type cells but not in NIk knockout cells (Fig. 6A,B). JNK is suggested to induce Raptor-S863 phosphorylation under strong hyperosmotic stress (Kwak et al. 2012). Consistently, we observed that, in N1k knockout cells, a high concentration of sorbitol could still increase Raptor-S863 phosphorylation, which was blocked by JNK inhibitor (Fig. 6C). These results show that Raptor-S863 can be phosphorylated by multiple kinases in response to different stress conditions, whereas NLK has a prominent role under mild osmotic stress.

To further study the function of Raptor S863 phosphorylation by NLK, we generated HEK293 cell lines bearing the Raptor-S863A or Raptor-S863D mutation using the CRISPR/Cas9 genome-editing system. The homozygous Raptor-S863A and Raptor-S863D knock-ins were confirmed by DNA sequencing (Supplemental Fig. S8A). No major defect in cell growth was observed in either cell line. Under normal culture conditions, cells with Raptor-S863A mutation showed as normal mTORC1 signaling (Fig. 6D). In contrast, the Raptor-S863D cells showed lower levels of S6K and 4EBP1 phosphorylation. However, the levels of Raptor-S863D protein as well as mTOR protein were reduced when compared with the wild-type cells (Fig. 6D). Since the mTOR protein was decreased, it was difficult to conclude whether the reduced phosphorylation of S6K and 4EBP1 was due to a decreased mTORC1 activation in the Raptor-S863D knock-in cells. It is worth noting that the Rag knockout cells also show a modest reduction, but not a complete loss, of mTORC1 activity (Jewell et al. 2015), results similar to the Raptor- 

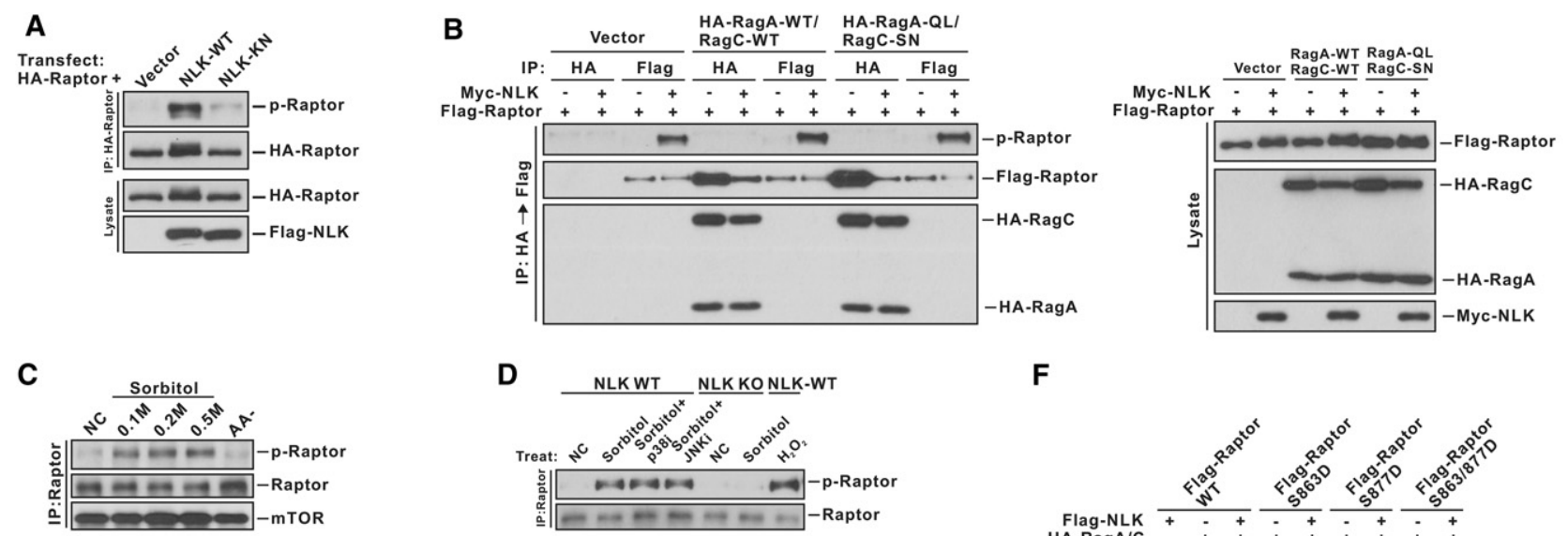

$\mathbf{F}$
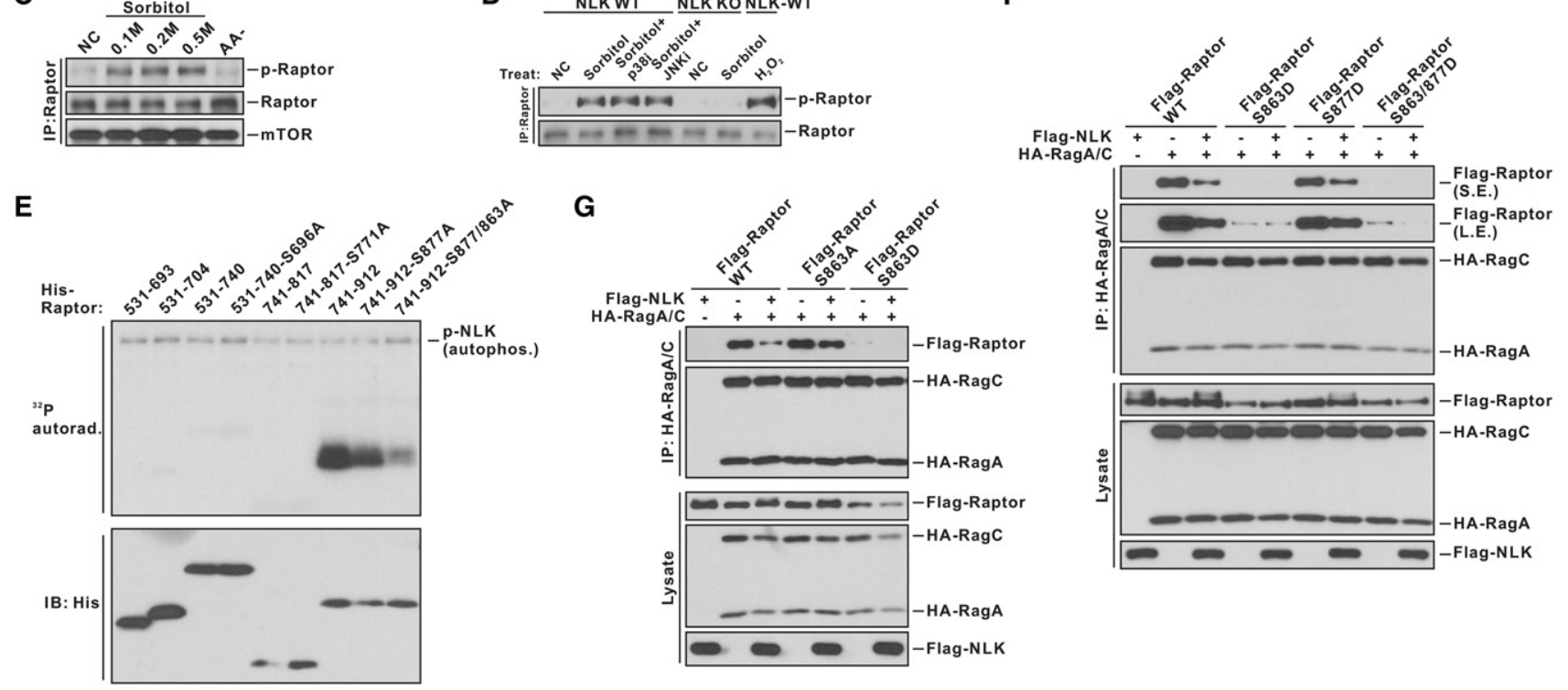

Figure 5. NLK phosphorylates Raptor at S863 in response to stress. (A) NLK induces Raptor phosphorylation on Thr-Pro and Ser-Pro motifs. Empty vector, NLK wild type (NLK-WT), or NLK-KN was transfected into HEK293 cells together with Raptor. NLK-induced phosphorylation of Raptor was detected with the antibody recognizing the p-Thr-Pro motif. $(B)$ The Raptor in complex with Rag is hypophosphorylated, while the Raptor not in complex with Rag is hyperphosphorylated. Empty vector or Myc-tagged NLK wild type was transfected together with Flag-tagged Raptor and HA-tagged RagA wild type/RagC wild type or RagA-QL/RagC-SN. Cell lysate was first subjected to immunoprecipitation with HA antibody to precipitate Rag complexes. The supernatant after HA antibody immunoprecipitation was then subjected to a secondary immunoprecipitation with Flag antibody to precipitate the remaining Raptor in the supernatant. To facilitate comparison of Raptor phosphorylation, Raptor from Flag immunoprecipitation was loaded at a level similar to that from HA co-IP. The right panel shows expression of Raptor, Rag, and NLK in the whole-cell lysate. $(C)$ Osmotic stress induces Raptor phosphorylation. HEK293 cells were treated with sorbitol at different concentrations for $15 \mathrm{~min}$ or starved with amino acid-free medium for 1 h. Endogenous Raptor was immunoprecipitated, and phosphorylation of Raptor at the p-Thr-Pro motif was detected with a specific antibody. $(D)$ NLK is required for Raptor phosphorylation by osmotic stress. Wild-type or Nlk knockout cells were in normal culture or were treated with $0.2 \mathrm{M}$ sorbitol or $0.25 \mathrm{mM} \mathrm{H}_{2} \mathrm{O}_{2}$ for $15 \mathrm{~min}$. Pretreatments with $10 \mu \mathrm{M}$ SB203580 (p38 inhibitor) or $20 \mu \mathrm{M}$ SP600125 (JNK inhibitor) are indicated. Following immunoprecipitation, phosphorylation of Raptor at the p-Thr-Pro motif was detected with a specific antibody. (E) S863 is a major NLK phosphorylation site in vitro. His-tagged Raptor fragments were purified and subjected to in vitro kinase assay with purified NLK kinase. Phosphorylation was detected by ${ }^{32} \mathrm{P}$-autoradiography. (F) The Raptor S863D mutant is defective in Rag interaction. Empty vector or NLK wild type was transfected with the Rag complex and wild-type Raptor or the Raptor S863D, Raptor S877D, or Raptor S863/877D mutant as indicated. The interaction between Raptor and Rag complexes was examined by co-IP. (G) The interaction between Raptor S863A and Rag is insensitive to inhibition by NLK. Empty vector or NLK wild type was transfected together with the Rag complex and wild-type Raptor or the Raptor S863A or Raptor S877D mutant as indicated. The interaction between Raptor and Rag complexes were examined by co-IP (see also Supplemental Fig. S7).

S863D cells, suggesting that cells have high plasticity in maintaining mTORCl activity.

We examined the integrity of $\mathrm{mTORC} 1$ and $\mathrm{mTORC} 2$ by immunoprecipitation of endogenous mTOR. When mTOR was normalized to similar levels, compatible Raptor and Rictor were detected in the mTOR immunoprecipitation from both wild-type and S863A cells. A mild decrease of the Raptor-S863D protein was found in
mTOR immunoprecipitation (Fig. 6E), correlating with the level of Raptor-S863D in cell lysate. These data indicate that mutation of Raptor S863, particularly S863A, does not significantly impair the integrity of $\mathrm{mTORC} 1$ or mTORC2.

Next, we tested the stress response of the Raptor phosphorylation mutant knock-in cell lines. Consistent with S863 being a major stress-inducible phosphorylation 
A

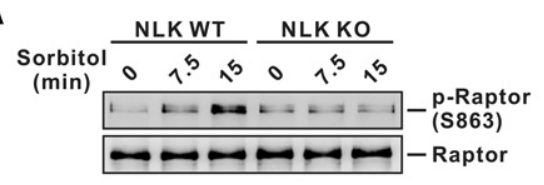

B

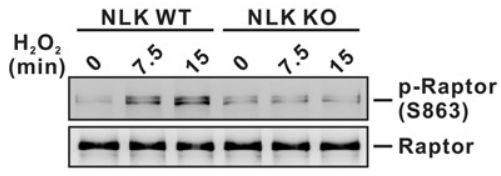

D

Cell Raptor Raptor Raptor line: WT S863A S863D

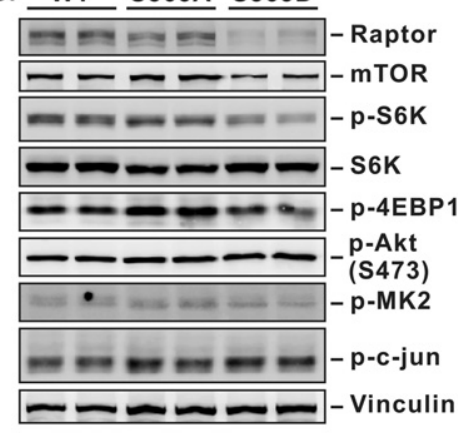

E

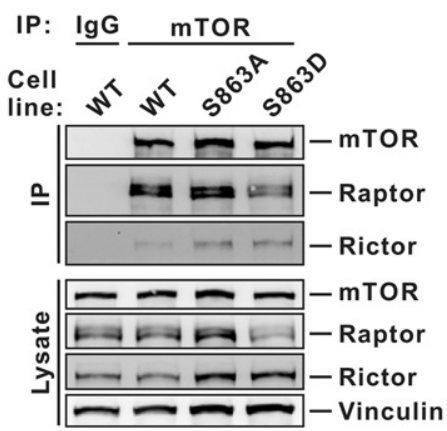

C

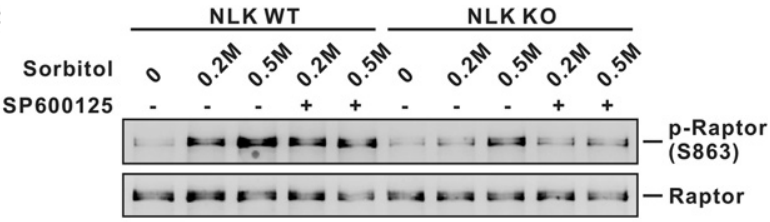

$\mathbf{F}$

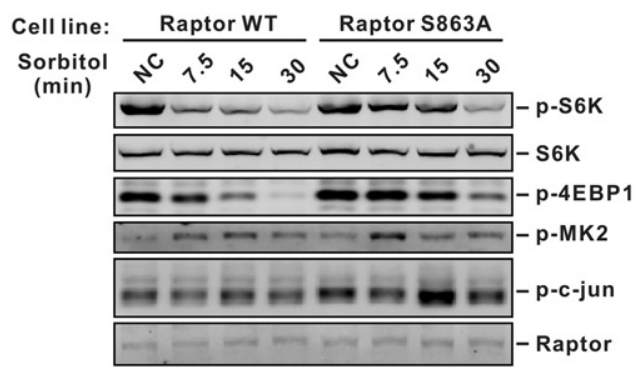

G

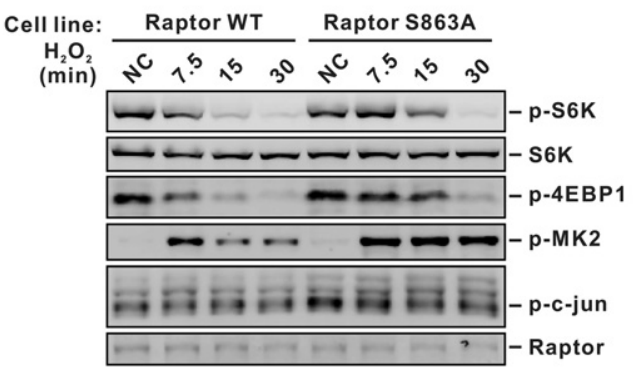

H

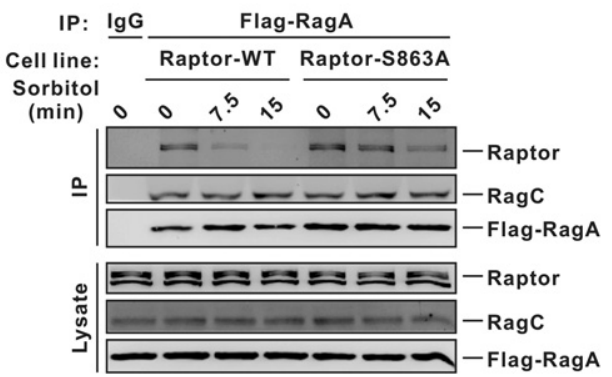

Figure 6. NLK inhibits mTORC1 by phosphorylating S863 in Raptor. (A) NLK is required for hyperosmotic stress-induced Raptor S863 phosphorylation. Wild-type or Nlk knockout cells were treated with $0.2 \mathrm{M}$ sorbitol at the indicated times. Endogenous Raptor was immunoprecipitated, and Raptor-S863 phosphorylation was detected with a specific antibody. $(B)$ NLK is required for oxidative stress-induced Raptor S863 phosphorylation. Experiments were similar to $A$ except cells were treated with $0.25 \mathrm{mM} \mathrm{H}_{2} \mathrm{O}_{2}$. (C) JNK is involved in Raptor S863 phosphorylation under severe hyperosmotic stress. As indicated, wild-type or Nlk knockout cells were treated with $0.2 \mathrm{M}$ or $0.5 \mathrm{M}$ sorbitol with or without pretreatment with JNK inhibitor (SP600125) for $30 \mathrm{~min}$. Phosphorylation of endogenous Raptor was determined as in A. (D) Characterization of Raptor S863A and S863D mutant cells. Cells were culture under nonstress conditions, and phosphorylation of mTORC1 substrates and other proteins was determined. (E) The mTORC1 and mTORC2 complex was not impaired in Raptor-S863 mutant cells. mTOR was immunoprecipitated from wild-type, S863A, or S863D cells. Raptor and Rictor levels in immunoprecipitation were examined with specific antibodies. $(F)$ Raptor S863A knock-in cells show compromised mTORC1 inhibition by osmotic stress. Cells containing wild-type or S863A Raptor were treated with $0.1 \mathrm{M}$ sorbitol for the times indicated. The activity of mTORC1, p38, and JNK was detected with specific antibodies. $(G)$ Raptor S863A knock-in cells show compromised mTORC1 inhibition by oxidative stress. Cells containing wild-type or S863A Raptor were treated with $0.25 \mathrm{mM} \mathrm{H}_{2} \mathrm{O}_{2}$ for the times indicated. The activity of mTORC1, p38, and JNK was detected with specific antibodies. $(H)$ Hyperosmotic stress fails to disrupt Raptor-Rag interaction in Raptor-S863A cells. Wild-type or Raptor-S863A cells stably expressing Flag-tagged RagA were treated with $0.2 \mathrm{M}$ sorbitol for the indicated times. The interaction between endogenous Raptor and ectoptically expressed Rag was examined by co-IP with Flag-RagA (see also Supplemental Fig. S8).

site, phosphorylation of Raptor-S863A was not induced by hyperosmotic stress or oxidative stress, as detected by the p-Thr-Pro antibody (Supplemental Fig. S8B). Furthermore, hyperosmotic stress-induced dephosphoryla- tion of S6K and 4EBP1 was delayed in the Raptor-S863A cells when compared with the wild-type cells (Fig. 6F). A similar defect in mTORC1 inhibition by oxidative stress was also observed in the Raptor-S863A cells (Fig. 6G). It 
should be noted that mTORC1 could still be inhibited by both osmotic stress and oxidative stress in the RaptorS863A knock-in cells, although the response was retarded. As expected, hyperosmotic stress could not effectively disrupt the interaction between Raptor and Rag in the Raptor-S863A knock-in cells (Fig. 6H). The above observations are similar to those found in the Nlk knockout cells (Fig. 2; Supplemental Fig. S3), indicating that Raptor S863 is a critical physiological phosphorylation site for NLK in mTORC1 regulation.

We tested whether S863 phosphorylation might play a role in modulating $\mathrm{mTOR}$ lysosomal localization in response to stress conditions. Both wild-type and RaptorS863A cells had normal mTOR speckles that were colocalized with lysosomes under normal culture (Fig. 7AC). Hyperosmotic stress rapidly abolished mTOR lysosomal localization in wild-type cells (Fig. 7A,C). In contrast, the osmotic stress-induced mTOR dissociation from lysosomes was significantly delayed in the RaptorS863A cells (Fig. 7B,C). These observations are consistent with the delayed mTORC1 inactivation in the RaptorS863A cells as well as in the Nlk knockout cells. These observations can be explained by the following model. In the absence of osmotic stress, amino acids activate Rag GTPases, which then bind to Raptor to recruit mTORC1 to lysosomes for activation. Hyperosmotic stress activates NLK, which phosphorylates Raptor S863, thereby inhibiting the Raptor-Rag interaction. Taken together, our results suggest that NLK mediates stress signals to inhibit mTORC1 through phosphorylating Raptor S863 residue and disrupting Rag-Raptor interaction (Fig. 7D). It should be noted that NLK is mainly responsible for the rapid osmotic effect on mTORC1, whereas other signaling pathways, such as JNK and p38, may be involved in the delayed osmotic response.

\section{Discussion}

As a central cell growth controller, mTORC1 has to integrate a wide range of both extracellular and intracellular

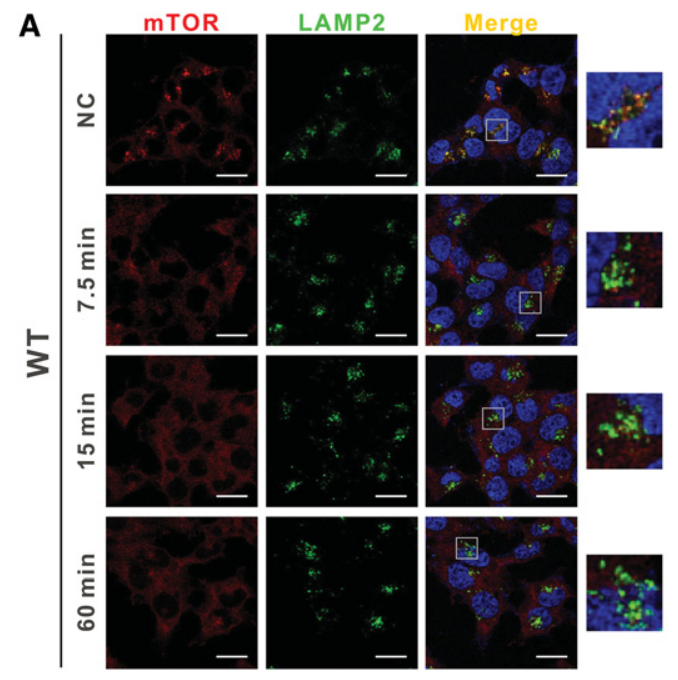

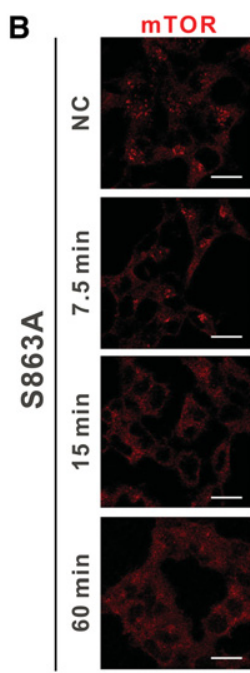
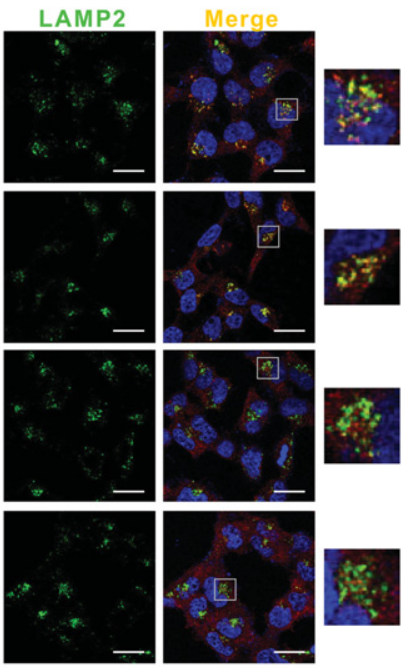

D (a)
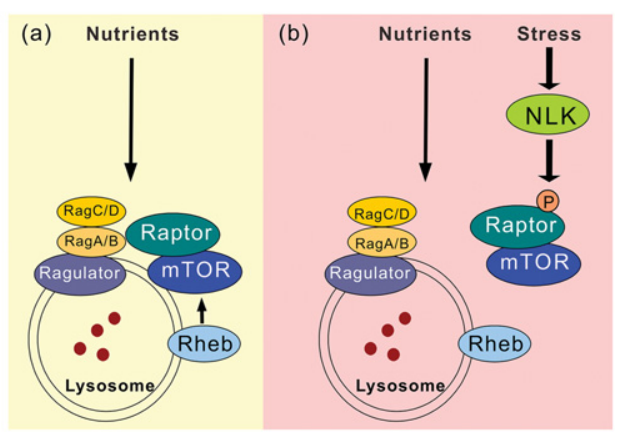

Figure 7. Raptor S863A mutation compromises stress-induced mTOR dissociation from lysosomes. $(A)$ Hyperosmotic stress rapidly induces $\mathrm{mTOR}$ dissociation from lysosomes. Wild-type cells were treated with sorbitol for the indicated times, followed by immunostaining with mTOR and LAMP2 antibodies. Magnified views are shown at the right. Bars, $10 \mu \mathrm{m} .(B)$ Raptor-S863A cells show a delayed mTOR dissociation from lysosomes. Experiments were similar to $A$ except Raptor-S863A cells were tested. (C) Quantification of mTOR dots that show colocalization with lysosome marker LAMP2 in $A$ and $B$. Twenty to 25 cells were randomly selected and counted. The asterisk indicates a statistically significant difference $(P<0.05)$. $(D)$ A proposed model of mTORC1 regulation by NLK. (Panel $a)$ Under nutrient-rich condition, the Rag complex is active and recruits mTORC1 to lysosomes through interaction with Raptor. $\mathrm{mTORC1}$ is then activated by lysosomally localized Rheb GTPase. (Panel $b$ ) When cells are under stress, NLK is activated and phosphorylates Raptor at the S863 site, which disrupts its interaction with the Rag complex. Therefore, mTORC1 dissociates with lysosome and is inactive. 
signals to regulate cell growth. The mechanisms of mTORC1 activation by growth factors and nutrients have been extensively investigated. How mTORC1 receives and integrates diverse signals, particularly inhibitory signals, to modulate cellular activity is a key question that has not be fully elucidated. In the present study, we discovered a new signaling mechanism involving NLK, a member of the atypical MAPK family. This study shows that NLK is important for the rapid MTORC1 inhibition by osmotic stress. Because NLK is capable of being activated through autophosphorylation (Ishitani et al. 2011), it is possible that NLK may be activated directly by stress conditions. NLK contains a unique N-terminal Ala-HisGln-rich domain and a C-terminal conserved Cys-rich domain (Brott et al. 1998); both domains are absent in other MAPKs. Further studies are needed to elucidate the mechanism of stress-induced NLK activation and whether these NLK-unique domains are involved.

We propose that NLK inhibits mTORC1 by disrupting its localization on lysosomes (Figs. 1B, 7D). mTORC1 was reported to be sequestered in stress granules upon stress, which served as a protective mechanism for cells under stress conditions (Takahara and Maeda 2012; Thedieck et al. 2013; Wippich et al. 2013). Based on previous reports and our data, one may speculate that phosphorylation of Raptor by NLK dissociates mTORC1 from lysosomes as well as facilitates mTORC1 translocation to stress granules in response to stress signals. Future study is needed to address this possibility.

Nlk knockout cells show normal mTORC1 activity under normal culture condition, suggesting that NLK takes part in mTORC1 regulation only when activated by stress. NIk deletion affects the rapid, but not the delayed, mTORC1 inhibition by osmotic stress. This indicates that, besides NLK, there are other mechanisms involved in mTORC1 inhibition at a late time point, indicating the complexity of cellular stress response. One may speculate a number of candidates that are involved in mTORC1 regulation by osmotic stress. For example, AMPK might be activated under stress to inhibit mTORC1 (Chen et al. 2010). Phosphatase activation upon hyperosmotic stress might also contribute to S6K dephosphorylation (Parrott and Templeton 1999; Kwak et al. 2012). Nevertheless, our result indicates that the compromised mTORC1 inhibition, not activation of phosphatase, is responsible for the altered S6K phosphorylation in the N1k knockout cells (Supplemental Fig. S4E).

Loss of NLK impairs cellular adaptation to mildly hypertonic conditions. It is notable that NLK-mediated mTORC1 inhibition is independent of the two wellknown stress-activated MAPKs p38 and JNK. Interestingly, p38 and JNK kinase show a compensatory activation when NIk is deleted, supporting the notion that NLK, p38, and JNK collectively modulate cell stress response. Consistent with this notion, partial inhibition of p38 and JNK enhanced the growth-inhibitory phenotype of the Nlk knockout cells by osmotic stress (Fig. 2D). Furthermore, inhibition of JNK abolished the residual Raptor S863 phosphorylation in the Nlk knockout cells (Fig. 6C). We reason that the Nlk knockout cells are defective in
mTORC1 inhibition and thus are unable to conserve energy and metabolites that are needed for proper stress response. Accordingly, N1k knockout cells showed higher protein synthesis than the control cells in the presence of sorbitol treatment (Fig. 2E,F). Although Nlk deletion only results in a short-term defect in $\mathrm{mTORC} 1$ regulation by osmotic stress, the Nlk knockout cells display a longterm effect, such as elevated p38 and JNK activation as well as reduced cell growth in response to stress. This suggests that a rapid stress response is critical for proper cellular adaptation.

Mechanistically, NLK inhibits mTORC1 through phosphorylating Raptor and disrupting its interaction with the Rag complex. Our results show that S863 of Raptor is the major site phosphorylated by NLK, and this phosphorylation mediates the NLK inhibition on mTORC1. The importance of S863 phosphorylation is demonstrated in the Raptor-S863A knock-in cells (Fig. 6F,G). RaptorS863D cells have a mild reduction in Raptor protein and mTORC1 activity (Fig. 6D). It is worth noting that the $\mathrm{RagA/B}$ knockout cells also have a modest reduction of mTORC1 activity (Jewell et al. 2015). These observations indicate strong plasticity/dynamics of cellular mTORC1 regulation. Cells find multiple ways to maintain a proper mTORC1 regulation.

Raptor S863 has been reported to be a common phosphorylation site by multiple kinases (Wang et al. 2009; Foster et al. 2010; Carriere et al. 2011; Wu et al. 2011; Kwak et al. 2012), including ERK1/2, JNK, p38 $\beta$, and mTOR. Therefore, Raptor S863 phosphorylation can occur in response to a number of stimuli. However, the functional significance of S863 phosphorylation is less clear. Expression of the Raptor S863A mutant is reported to cause a mild decrease of mTORC1 activity, indicating that phosphorylation of S863 might positively contribute to mTORC1 activity (Foster et al. 2010; Wu et al. 2011). A caveat of previous studies is that these functional assays were performed with ectopic expression of Raptor. It is worth noting that overexpression of wild-type Raptor inhibits mTORC1 activity (Kim et al. 2002), possibly by interfering with the mTORC1 complex or blocking the Ragdependent recruitment of MTORC1 to lysosomes due to the excessive free Raptor protein. Therefore, previous studies performed with the Raptor S863A overexpression should be interpreted with caution.

We addressed the functional significance of Raptor S863 phosphorylation by creating Raptor S863A and S863D knock-in cells. The expression level of Ratpor-S863A is indistinguishable from that of wild-type Raptor, while the expression of Raptor-S863D is slightly lower. Our data clearly show that Raptor-S863A does not reduce basal mTORC1 activity. Instead, the Raptor S863A knock-in cells show a delayed mTORC1 inhibition in response to osmotic stress and oxidative stress. Consistently, the Raptor-S863A cells also show a delay of mTOR dissociation from lysosomes under hyperosmotic stress. Consistent with the functional data, our biochemical data show that Raptor S863 phosphorylation disrupts its interaction with Rag but not mTOR. Collectively, our study provides a molecular model explaining how stress inhibits 
mTORC1 via NLK (Fig. 7D). Our study also explains why the effect of osmotic stress on mTORC1 is dominant over the positive signals of growth factors or nutrients because NLK acts directly on Raptor, while both growth factors and nutrients act upstream of mTORC1. In summary, this study reveals NLK as a novel stress sensor kinase that mediates $\mathrm{mTORC} 1$ inhibition and therefore addresses a key missing step in mTORCl regulation: how mTORC1 integrates stress signals with growth-stimulating signals to produce a coordinated cellular response.

\section{Materials and methods}

\section{Purification of NLK kinase from mammalian cells}

pLVX-GST-NLK-Flag was transiently transfected into HEK293 cells. After $44 \mathrm{~h}$, cells were treated with $0.2 \mathrm{M}$ sorbitol for $15 \mathrm{~min}$ and lysed in Triton lysis buffer as described above. Cell lysate was sonicated at mild power for $15 \mathrm{sec}$ and cleared by centrifugation. NLK kinase was purified by a two-step protocol. First, cell lysate was incubated with anti-Flag M2 affinity gel (Sigma, A2220) for $3 \mathrm{~h}$ at $4^{\circ} \mathrm{C}$. Beads were washed three times for $5 \mathrm{~min}$ with lysis buffer (0.5 M NaCl was added for the last wash). Flagtagged protein was eluted with $200 \mu \mathrm{g} / \mathrm{mL} 3 \times$ Flag peptide (diluted in lysis buffer) for $60 \mathrm{~min}$ at $4^{\circ} \mathrm{C}$. For the secondary purification, glutathione (GSH)-Sepharose beads (GE, 17-0756-01) were added to the eluted Flag protein solution and incubated with rotation for $1.5 \mathrm{~h}$ at $4^{\circ} \mathrm{C}$. Beads were then washed three times with lysis buffer and once with elution buffer $(20 \mathrm{mM}$ Tris at $\mathrm{pH}$ $8.0,100 \mathrm{mM} \mathrm{NaCl}$ ). GST-tagged protein was eluted by incubation with elution buffer containing $20 \mathrm{mM}$ reduced GSH for $30 \mathrm{~min}$ at $4^{\circ} \mathrm{C}$. Eluted protein was dialyzed in the kinase stocking buffer (20 mM MOPS-NaOH at pH 7.0, 1 mM EDTA, 0.01\% Brij-35, $5 \%$ glycerol, $0.1 \%$ 2-mercaptoethanol, $1 \mathrm{mg} / \mathrm{ml} \mathrm{BSA}$ ) overnight.

\section{In vitro kinase assay}

The in vitro kinase assay for NLK activation or its phosphorylation on Raptor fragments was described previously (Ishitani et al. 2009). Briefly, for the NLK activation assay, HEK293 cells stably expressing HA-tagged NLK were treated as indicated in the figures. HA-NLK was then immunoprecipitated from cells and subjected to in vitro kinase assay using GST-LEF1-51-210 as a substrate. Reactions were performed in kinase buffer (10 mM Hepes at pH 7.4, 1 mM DTT, $5 \mathrm{mM} \mathrm{MgCl}_{2}, 5 \mu \mathrm{Ci}\left[{ }^{32} \mathrm{P}\right]$ ATP) for $2.5 \mathrm{~min}$ at room temperature. For the Raptor phosphorylation assay, purified NLK kinase was mixed with 300 ng of Raptor fragment proteins in kinase buffer supplemented with $50 \mu \mathrm{M}$ ATP and incubated for $30 \mathrm{~min}$ at $30^{\circ} \mathrm{C}$. The samples were resolved by SDS-PAGE, and phosphorylated proteins were visualized by autoradiography.

\section{Cell growth assay}

Equal amounts of wild-type or N1k knockout cells $\left(6 \times 10^{5}\right)$ were plated in 60-mm dishes. One set of plates was treated with trypsin after $2 \mathrm{~h}$ when cells just attached to plates. Cells were counted, and the numbers were set for zero point. Cells in other dishes were incubated in normal medium or medium containing 0.1 M sorbitol, $2 \mu$ M SB203580/4 $\mu$ M SP600125, or both sorbitol and SB203580/SP600125. Media and supplements were replenished every $24 \mathrm{~h}$. Cells were trypsinized at different time points and counted by using Countstar IC1000. Experiments were repeated three times.

\section{Protein synthesis assay}

Wild-type or NIk knockout cells were washed with PBS and incubated in methionine and cysteine-free DMEM with or without $0.1 \mathrm{M}$ sorbitol for $5 \mathrm{~min} .{ }^{35} \mathrm{~S}$-labeled L-methionine and L-cysteine $\operatorname{mix}(75 \mu \mathrm{Ci}$ in a $35-\mathrm{mm}$ dish) (PerkinElmer, NEG772014MC) was then added to the medium, and cells were incubated for an additional $10 \mathrm{~min}$. Cells were quickly washed with cold PBS and lysed with sample buffer. Proteins were resolved by SDS-PAGE, and new synthesized proteins were detected by autoradiography. Densitometric analysis of each lane as well as vinculin was performed with Quantity One software (Bio-Rad). Experiments were repeated three times, and data were shown as the ratio of the densitometric level with sorbitol treatment to that without sorbitol treatment after normalization to vinculin.

\section{Generation of RagA and Nlk knockout cells using CRISPR/Cas9 genome editing}

Generation of RagA knockout HEK293A cells was described before (Jewell et al. 2015), and the protocol was followed for the generation of Nlk knockout HEK293 cells. The two 20-nucleotide (nt) guide sequences targeting exon 1 of the human Nlk gene were sequence 1, 5' -AAAATGATGGCGGCTTACAA-3' (clones 1-8), and sequence 2, 5'-ACACCATCTTCATCCGGGGT-3' (clones 2-12). To generate Nlk knockout Neuro-2a cells, the following guide sequence targeting exon 1 of mouse Nlk gene was used: 5'-AAAATGATGGCGGCTTACAA-3'.

Guide sequences were cloned into a bicistronic expression vector (pX330) containing human codon-optimized Cas9 and the RNA components (Hsu et al. 2013). HEK293 cell lines with Nlk knockout were screened and verified by both Western blot for whole-cell lysate and Sanger sequencing of genomic DNA (Supplemental Fig. S2).

Generation of HEK293 cell lines bearing a Raptor S863A or S863D mutation using CRISPR/Cas9 genome editing

The protocol to generate Raptor mutant cells was similar to the one for generating Nlk knockout cells except a donor plasmid containing Raptor genomic sequence bearing the S863A or S863D mutation was cotransfected with the Cas9 construct. The 20-nt guide sequences targeting the genomic sequence near the S863 site of the human Raptor gene (shown below) were cloned into pX459 vector (5'-TGGCGGGGGCCGACTGCG TG-3'). The donor plasmids were made in three steps: (1) A 1705-base-pair (bp) genomic sequence from the human Raptor gene was amplified with the following primers and subcloned into pRK7 vector (forward, 5'-GTGGGAAAGGATGACCACA G-3'; reverse, 5'-GAACTGGTGGGAGTGAGGG-3'). (2) We introduced a synonymous mutation of the last " $\mathrm{G}$ " in the PAM (NGG) to "C" to prevent recognition and digestion of the donor by Cas9. Primers for mutation were forward, 5'-GGA CACCTCCTCGCTCACGCAGTCG - $3^{\prime}$, and reverse, $5^{\prime}$-CGACT GCGTGAGCGAGGAGGTGTCC-3'. (3) The S863 site was mutated to either alanine (A) or aspartic acid (D). Primers for the S863A mutation were forward, 5'-GTCGGCCCCCGCCGCC CCCACCAACAAG-3', and reverse, 5'-CTTGTTGGTGGGGG CGGCGGGGGCCGAC-3'. Primers for the S863D mutation were forward, 5'-GTCGGCCCCCGCCGACCCCACCAACA AG-3', and reverse, 5'-CTTGTTGGTGGGGTCGGCGGGGGC CGAC- $3^{\prime}$.

HEK293 cell lines with a Raptor S863A or S863D mutation were screened and verified by Sanger sequencing of the genomic DNA (Supplemental Fig. S5C). 


\section{Statistical analysis}

Statistical significance of differences between mean values $(P<0.05)$ was evaluated using the unpaired Student's $t$-test. All data were expressed as the mean \pm standard deviation.

\section{Acknowledgments}

This work was supported by grants from the National Health Institutes (R01GM51586 and R35CA196878 to K.-L.G., and T32CA121938 to J.L.J.) and U.S. Department of Defense (W81XWH-13-055 to K.-L.G.). This work was also supported by a National Natural Science Foundation of China grant (no. 31570784 to H.-X.Y.). R.C.R. was supported by a Canadian Institutes of Health Research (CIHR) post-doctoral fellowship.

\section{References}

Barnes $\mathrm{K}$, Ingram JC, Porras OH, Barros LF, Hudson ER, Fryer LG, Foufelle F, Carling D, Hardie DG, Baldwin SA. 2002. Activation of GLUT1 by metabolic and osmotic stress: potential involvement of AMP-activated protein kinase (AMPK). J Cell Sci 115: 2433-2442.

Bar-Peled L, Schweitzer LD, Zoncu R, Sabatini DM. 2012. Ragulator is a GEF for the rag GTPases that signal amino acid levels to mTORC1. Cell 150: 1196-1208.

Bar-Peled L, Chantranupong L, Cherniack AD, Chen WW, Ottina KA, Grabiner BC, Spear ED, Carter SL, Meyerson M, Sabatini DM. 2013. A Tumor suppressor complex with GAP activity for the Rag GTPases that signal amino acid sufficiency to mTORC1. Science 340: 1100-1106.

Brott BK, Pinsky BA, Erikson RL. 1998. Nlk is a murine protein kinase related to Erk/MAP kinases and localized in the nucleus. Proc Natl Acad Sci 95: 963-968.

Burg MB, Ferraris JD, Dmitrieva NI. 2007. Cellular response to hyperosmotic stresses. Physiol Rev 87: 1441-1474.

Carriere A, Romeo Y, Acosta-Jaquez HA, Moreau J, Bonneil E, Thibault P, Fingar DC, Roux PP. 2011. ERK1/2 phosphorylate Raptor to promote Ras-dependent activation of mTOR complex 1 (mTORC1). J Biol Chem 286: 567-577.

Chen D, Fucini RV, Olson AL, Hemmings BA, Pessin JE. 1999. Osmotic shock inhibits insulin signaling by maintaining Akt/protein kinase B in an inactive dephosphorylated state. Mol Cell Biol 19: 4684-4694.

Chen L, Xu B, Liu L, Luo Y, Yin J, Zhou H, Chen W, Shen T, Han X, Huang S. 2010. Hydrogen peroxide inhibits mTOR signaling by activation of AMPKa leading to apoptosis of neuronal cells. Lab Invest 90: 762-773.

Choi KW, Benzer S. 1994. Rotation of photoreceptor clusters in the developing Drosophila eye requires the nemo gene. Cell 78: $125-136$.

Foster KG, Acosta-Jaquez HA, Romeo Y, Ekim B, Soliman GA, Carriere A, Roux PP, Ballif BA, Fingar DC. 2010. Regulation of mTOR complex 1 (mTORC1) by raptor Ser863 and multisite phosphorylation. J Biol Chem 285: 80-94.

Han J, Lee JD, Bibbs L, Ulevitch RJ. 1994. A MAP kinase targeted by endotoxin and hyperosmolarity in mammalian cells. Science 265: 808-811.

Hawley SA, Ross FA, Chevtzoff C, Green KA, Evans A, Fogarty S, Towler MC, Brown LJ, Ogunbayo OA, Evans AM, et al. 2010. Use of cells expressing $\gamma$ subunit variants to identify diverse mechanisms of AMPK activation. Cell Metab 11: 554-565.

Hohmann S, Krantz M, Nordlander B. 2007. Yeast osmoregulation. Methods Enzymol 428: 29-45.
Hsu PD, Scott DA, Weinstein JA, Ran FA, Konermann S, Agarwala V, Li Y, Fine EJ, Wu X, Shalem O, et al. 2013. DNA targeting specificity of RNA-guided Cas9 nucleases. Nat Biotechnol 31: 827-832.

Inoki K, Li Y, Zhu T, Wu J, Guan KL. 2002. TSC2 is phosphorylated and inhibited by Akt and suppresses mTOR signalling. Nat Cell Biol 4: 648-657.

Inoki K, Li Y, Xu T, Guan KL. 2003a. Rheb GTPase is a direct target of TSC2 GAP activity and regulates mTOR signaling. Genes Dev 17: 1829-1834.

Inoki K, Zhu T, Guan KL. 2003b. TSC2 mediates cellular energy response to control cell growth and survival. Cell 115: 577-590.

Ishitani T, Ninomiya-Tsuji J, Nagai S, Nishita $M$, Meneghini $M$, Barker N, Waterman M, Bowerman B, Clevers H, Shibuya $H$, et al. 1999. The TAK1-NLK-MAPK-related pathway antagonizes signalling between $\beta$-catenin and transcription factor TCF. Nature 399: 798-802.

Ishitani T, Kishida S, Hyodo-Miura J, Ueno N, Yasuda J, Waterman M, Shibuya H, Moon RT, Ninomiya-Tsuji J, Matsumoto K. 2003a. The TAK1-NLK mitogen-activated protein kinase cascade functions in the Wnt-5a/Ca ${ }^{2+}$ pathway to antagonize Wnt/ $\beta$-catenin signaling. Mol Cell Biol 23: 131-139.

Ishitani T, Ninomiya-Tsuji J, Matsumoto K. 2003b. Regulation of lymphoid enhancer factor $1 / \mathrm{T}$-cell factor by mitogen-activated protein kinase-related Nemo-like kinase-dependent phosphorylation in Wnt/ $\beta$-catenin signaling. Mol Cell Biol 23: 1379-1389.

Ishitani T, Ishitani S, Matsumoto K, Itoh M. 2009. Nemo-like kinase is involved in NGF-induced neurite outgrowth via phosphorylating MAP1B and paxillin. I Neurochem 111: 1104-1118.

Ishitani T, Hirao T, Suzuki M, Isoda M, Ishitani S, Harigaya $\mathrm{K}$, Kitagawa M, Matsumoto K, Itoh M. 2010. Nemo-like kinase suppresses Notch signalling by interfering with formation of the Notch active transcriptional complex. Nat Cell Biol 12: $278-285$.

Ishitani S, Inaba K, Matsumoto K, Ishitani T. 2011. Homodimerization of Nemo-like kinase is essential for activation and nuclear localization. Mol Biol Cell 22: 266-277.

Jewell JL, Kim YC, Russell RC, Yu FX, Park HW, Plouffe SW, Tagliabracci VS, Guan KL. 2015. Metabolism. Differential regulation of mTORC1 by leucine and glutamine. Science 347 : 194-198.

Kanei-Ishii C, Ninomiya-Tsuji J, Tanikawa J, Nomura T, Ishitani T, Kishida S, Kokura K, Kurahashi T, Ichikawa-Iwata E, Kim $\mathrm{Y}$, et al. 2004. Wnt-1 signal induces phosphorylation and degradation of c-Myb protein via TAK1, HIPK2, and NLK. Genes Dev 18: 816-829.

Kim DH, Sarbassov DD, Ali SM, King JE, Latek RR, ErdjumentBromage H, Tempst P, Sabatini DM. 2002. mTOR interacts with raptor to form a nutrient-sensitive complex that signals to the cell growth machinery. Cell 110: 163-175.

Kim E, Goraksha-Hicks P, Li L, Neufeld TP, Guan KL. 2008. Regulation of TORC1 by Rag GTPases in nutrient response. Nat Cell Biol 10: 935-945.

Kojima H, Sasaki T, Ishitani T, Iemura S, Zhao H, Kaneko S, Kunimoto H, Natsume T, Matsumoto K, Nakajima K. 2005. STAT3 regulates Nemo-like kinase by mediating its interaction with IL-6-stimulated TGF $\beta$-activated kinase 1 for STAT3 Ser-727 phosphorylation. Proc Natl Acad Sci 102: 4524-4529.

Kwak D, Choi S, Jeong H, Jang JH, Lee Y, Jeon H, Lee MN, Noh J, Cho K, Yoo JS, et al. 2012. Osmotic stress regulates mammalian target of rapamycin (mTOR) complex 1 via c-Jun $\mathrm{N}$ - 
terminal kinase (JNK)-mediated Raptor protein phosphorylation. J Biol Chem 287: 18398-18407.

Laplante M, Sabatini DM. 2012. mTOR signaling in growth control and disease. Cell 149: 274-293.

Ohkawara B, Shirakabe K, Hyodo-Miura J, Matsuo R, Ueno N, Matsumoto K, Shibuya H. 2004. Role of the TAK1-NLKSTAT3 pathway in TGF- $\beta$-mediated mesoderm induction. Genes Dev 18: 381-386.

Ortells MC, Morancho B, Drews-Elger K, Viollet B, Laderoute KR, Lopez-Rodriguez C, Aramburu J. 2012. Transcriptional regulation of gene expression during osmotic stress responses by the mammalian target of rapamycin. Nucleic Acids Res 40: 4368-4384.

Ota S, Ishitani S, Shimizu N, Matsumoto K, Itoh M, Ishitani T. 2012. NLK positively regulates $\mathrm{Wnt} / \beta$-catenin signalling by phosphorylating LEF1 in neural progenitor cells. EMBO J 31: 1904-1915.

Parrott LA, Templeton DJ. 1999. Osmotic stress inhibits p70/85 S6 kinase through activation of a protein phosphatase. I Biol Chem 274: 24731-24736.

Sancak Y, Peterson TR, Shaul YD, Lindquist RA, Thoreen CC, Bar-Peled L, Sabatini DM. 2008. The Rag GTPases bind raptor and mediate amino acid signaling to mTORC1. Science 320: 1496-1501.

Sancak Y, Bar-Peled L, Zoncu R, Markhard AL, Nada S, Sabatini DM. 2010. Ragulator-Rag complex targets MTORC1 to the lysosomal surface and is necessary for its activation by amino acids. Cell 141: 290-303.

Sarbassov DD, Guertin DA, Ali SM, Sabatini DM. 2005. Phosphorylation and regulation of Akt/PKB by the rictor-mTOR complex. Science 307: 1098-1101.

Saucedo LJ, Gao X, Chiarelli DA, Li L, Pan D, Edgar BA. 2003. Rheb promotes cell growth as a component of the insulin/ TOR signalling network. Nat Cell Biol 5: 566-571.
Sengupta S, Peterson TR, Sabatini DM. 2010. Regulation of the mTOR complex 1 pathway by nutrients, growth factors, and stress. Mol Cell 40: 310-322.

Smit L, Baas A, Kuipers J, Korswagen H, van de Wetering M, Clevers H. 2004. Wnt activates the Tak1/Nemo-like kinase pathway. J Biol Chem 279: 17232-17240.

Stocker H, Radimerski T, Schindelholz B, Wittwer F, Belawat $\mathrm{P}$, Daram P, Breuer S, Thomas G, Hafen E. 2003. Rheb is an essential regulator of $\mathrm{S} 6 \mathrm{~K}$ in controlling cell growth in Drosophila. Nat Cell Biol 5: 559-565.

Takahara T, Maeda T. 2012. Transient sequestration of TORC1 into stress granules during heat stress. Mol Cell 47: 242-252.

Thedieck K, Holzwarth B, Prentzell MT, Boehlke C, Klasener K, Ruf S, Sonntag AG, Maerz L, Grellscheid SN, Kremmer E, et al. 2013. Inhibition of mTORC1 by astrin and stress granules prevents apoptosis in cancer cells. Cell 154: 859-874.

Thorpe CJ, Moon RT. 2004. nemo-like kinase is an essential coactivator of Wnt signaling during early zebrafish development. Development 131: 2899-2909.

Wang L, Lawrence JC Jr, Sturgill TW, Harris TE. 2009. Mammalian target of rapamycin complex 1 (mTORC1) activity is associated with phosphorylation of raptor by mTOR. J Biol Chem 284: 14693-14697.

Whitmarsh AJ. 2010. A central role for p38 MAPK in the early transcriptional response to stress. BMC Biol 8: 47.

Wippich F, Bodenmiller B, Trajkovska MG, Wanka S, Aebersold R, Pelkmans L. 2013. Dual specificity kinase DYRK3 couples stress granule condensation/dissolution to mTORC1 signaling. Cell 152: 791-805.

Wu XN, Wang XK, Wu SQ, Lu J, Zheng M, Wang YH, Zhou H, Zhang H, Han J. 2011. Phosphorylation of Raptor by $\mathrm{p} 38 \beta$ participates in arsenite-induced mammalian target of rapamycin complex 1 (mTORC1) activation. I Biol Chem 286: 31501-31511. 


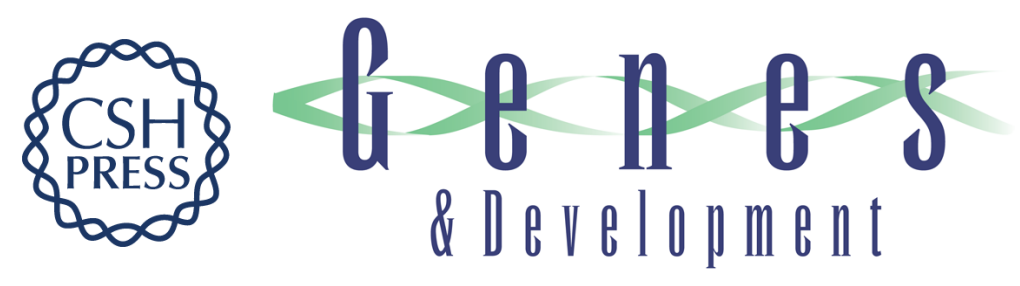

\section{NLK phosphorylates Raptor to mediate stress-induced mTORC1 inhibition}

Hai-Xin Yuan, Zhen Wang, Fa-Xing Yu, et al.

Genes Dev. 2015, 29:

Access the most recent version at doi:10.1101/gad.265116.115

\section{Supplemental http://genesdev.cshlp.org/content/suppl/2015/11/19/29.22.2362.DC1 Material}

References This article cites 48 articles, 24 of which can be accessed free at: http://genesdev.cshlp.org/content/29/22/2362.full.html\#ref-list-1

Creative This article is distributed exclusively by Cold Spring Harbor Laboratory Press for the first Commons six months after the full-issue publication date (see

License http://genesdev.cshlp.org/site/misc/terms.xhtml). After six months, it is available under a Creative Commons License (Attribution-NonCommercial 4.0 International), as described at http://creativecommons.org/licenses/by-nc/4.0/.

Email Alerting Receive free email alerts when new articles cite this article - sign up in the box at the top Service right corner of the article or click here.

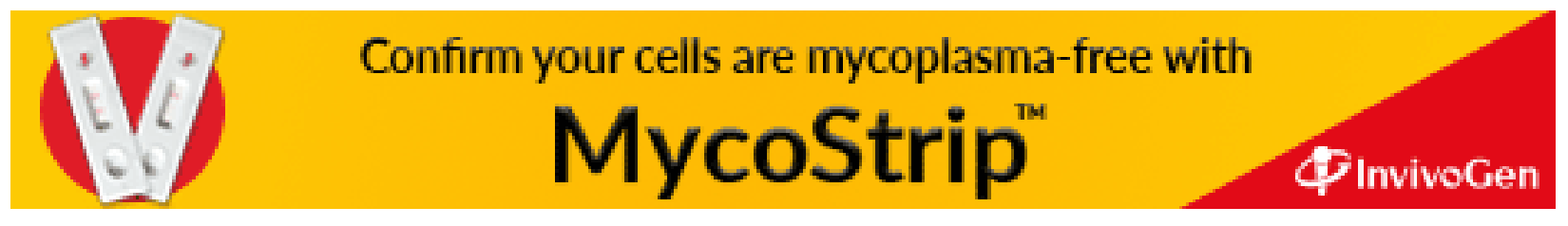

